\title{
Variance-Constrained Recursive State Estimation for Time-Varying Complex Networks with Quantized Measurements and Uncertain Inner Coupling
}

\author{
Jun $\mathrm{Hu}$, Member, IEEE, Zidong Wang, Fellow, IEEE, Guo-Ping Liu, Fellow, IEEE and Hongxu Zhang
}

\begin{abstract}
In this paper, a new recursive state estimation problem is discussed for a class of discrete time-varying stochastic complex networks with uncertain inner coupling and signal quantization under the error-variance constraints. The coupling strengths are allowed to be varying within certain intervals, and the measurement signals are subject to quantization effects before being transmitted to the remote estimator. The focus of the conducted topic is on the design of a variance-constrained state estimation algorithm with aim to ensure a locally minimized upper bound on the estimation error covariance at every sampling instant. Furthermore, the boundedness of the resulting estimation error is analyzed and a sufficient criterion is established to ensure the desired exponential boundedness of the state estimation error in mean square sense. Finally, some simulations are proposed with comparisons to illustrate the validity of newly developed variance-constrained estimation method.
\end{abstract}

Index Terms-Time-varying stochastic complex networks, optimal state estimation, signal quantization, uncertain inner coupling, boundedness analysis, variance-constrained approach.

\section{INTRODUCTION}

$\mathbf{C}$ OMPLEX networks characterize the dynamical networks consisted of plenty of interacted dynamical elements, which can be found in many real-world networks such as technological networks, power grid networks, computer networks, biological networks and social networks [4], [22]. Over the past decade, there has been a surge of research into the analysis problems of complex dynamical networks due primarily to their successful applications [9], [26], [36]. In general, it is of theoretical importance to obtain the state information of

This work was supported in part by the National Natural Science Foundation of China under Grants 61673141, 61873148 and 61773144, the Outstanding Youth Science Foundation of Heilongjiang Province of China under grant JC2018001, the Fok Ying Tung Education Foundation of China under Grant 151004, and the Alexander von Humboldt Foundation of Germany.

$\mathrm{J}$. $\mathrm{Hu}$ is with the School of Engineering, University of South Wales, Pontypridd CF37 1DL, United Kingdom, and also with the Department of Mathematics, Harbin University of Science and Technology, Harbin 150080, China. (Email: hujun2013@gmail.com)

Z. Wang is with the Department of Computer Science, Brunel University London, Uxbridge, Middlesex, UB8 3PH, United Kingdom. (Email: Zidong. Wang@brunel.ac.uk)

G. Liu is with the School of Engineering, University of South Wales, Pontypridd CF37 1DL, United Kingdom, and also with the Department of Artificial Intelligence and Automation, Wuhan University, Wuhan 430072, China. (Email: guoping. liuesouthwales.ac.uk)

H. Zhang is with the School of Measurement Control Technology \& Communication Engineering, Harbin University of Science and Technology, Harbin 150080, Chinam and also with the Heilongjiang Provincial Key Laboratory of Optimization Control and Intelligent Analysis for Complex Systems, Harbin University of Science and Technology, Harbin 150080 China. (Email: hongxuzhang@hrbust.edu.cn) the network nodes so as to better understand the intrinsic structures and dynamical behaviors of complex networks [5], [43]. However, for network nodes, it is quite common in reality that the accurate state information of the network nodes is inaccessible for various reasons (e.g. limited resources and harsh environments), and only the measurement outputs of the network nodes are made available [1], [3], [6], [7], [14], [37], [40]. As such, the state estimation problems under different indices for complex dynamical networks have recently received considerable research attention and a large number of state estimation algorithms have been proposed according to specific performance requirements [11], [22], [26], [29], [30]. For example, the asynchronous state estimation method with guaranteed dissipativity has been developed in [38] for stochastic complex networks subject to uncertain measurements described by a multiplicative noise. To attenuate the impacts from energy-bounded disturbances, in [11], [29], [30], the $H_{\infty}$ state estimation algorithms have been put forward through available measurement outputs of stochastic complex networks. Very recently, the recursive state estimation scheme under variance constraint has been presented in [15] for a class of time-varying networked complex networks with stochastic noises, where the effects from the missing measurements onto the state estimation algorithm accuracy have been thoroughly discussed. Subsequently, based on [15], the state estimator under the variance constraint has been designed in [21] for timevarying nonlinear complex dynamical networks with stochastic noises.

As is well known, the nodes of a complex network are intrinsically coupled under a given topological structure which, in turn, affects the dynamical behaviors of the network itself. In the context of node coupling, most existing results have been based on the hypothesis that the coupling strengths between the network nodes are precisely known [42]. Such an assumption is, unfortunately, fairly restrictive in certain practical applications. For example, it has been pointed out in [18], [24], [29] that the coupling strengths could be uncertain or even unavailable in engineering practice. Accordingly, some efforts have been devoted to the synchronization/estimation problems for many complex dynamical networks subject to uncertain inner couplings with some excellent results appeared in the literature [20], [27], [29], [32]. For example, the exponential synchronization criterion has been given in [27] for Lur'e complex networks subject to uncertain coupling strength by designing a pinning impulsive controller. The synchronization conditions have been presented in [20], [32] for delayed 
complex networks with uncertain inner couplings, where the event-triggered control mechanisms have been employed to reduce the utilizations of the limited communication resources. Regarding the state estimation problems, a robust $H_{\infty}$ state estimation approach has been developed in [29] for discrete complex networks with imperfect measurements characterized by the Kronecker delta function. Nevertheless, all the aforementioned results concerning uncertain inner couplings are only applicable to time-invariant complex networks, and there is a practical need to look into the design problem of the state estimator for time-varying stochastic complex networks, where new methodologies ought to be sought to tackle the time-varying feature.

In nowadays pervasive networked environments, the measurement signals are often quantized before the transmission due mainly to the limited capacities of the communication channels [2], [10], [19], [25], [31], [39]. In [34], the way the quantization affects the network properties has been examined by utilizing the spectrum of the Laplacian of the underlying complex network. Recently, the synchronization and state estimation problems have received some initial research attention for complex networks with uniform/logarithmic quantization. For instance, the global synchronization criterion has been derived in [33] for complex networks under quantization effects, where the uniform quantizer has been employed during the digital communications and a scaling function has been used to ensure the bounded quantization levels. With respect to the logarithmic quantization, the asynchronous $H_{\infty}$ state estimation algorithm has been designed in [41] for discretetime switched dynamical networks subject to packet dropouts, event-triggered mechanism as well as quantization effects. The event-based state estimation approach has been proposed in [23] for complex network systems with quantization effects and randomly switching nonlinearities. Unfortunately, to the best of our knowledge, the recursive state estimation problem for discrete time-varying stochastic complex networks subject to quantized measurements has not received adequate attention, not to mention the case that both the uncertain inner coupling and the variance constraints are also addressed. Consequently, the main motivation of the paper is to shorten such a gap by developing a new variance-constrained state estimation algorithm.

Motivated by the above analysis, we aim to address the variance-constrained recursive state estimation problem for a class of time-varying stochastic complex networks subject to uncertain inner coupling and quantization effects. Firstly, the uncertain inner coupling with known upper and lower bounds is considered in the system modeling, and the quantization errors induced by the logarithmic quantization are addressed simultaneously. Secondly, the phenomena of uncertain coupling and quantization effects are thoroughly investigated, and a computationally appealing algorithm is developed to recursively estimate the network states where the information about the coupling strength bounds and the quantization levels are explicitly employed. A rigorous stochastic analysis is carried out to guarantee the existence of an upper bound on the error variance of the state estimation at each time step and such an obtained upper bound is subsequently minimized. A further analysis is conducted on the boundedness issue of the estimation errors to quantify the performance of the proposed state estimation algorithm. Finally, some simulations under different quantization effects are performed to illustrate the validity of the variance-constrained state estimation approach proposed in the paper.

The major contributions of the main results are outlined as follows: 1) the effects from both the uncertain inner coupling and the quantization errors are tackled simultaneously when handling the variance-constrained state estimation problem for discrete time-varying stochastic complex networks; 2) the locally optimal state estimation method under variance constraint is developed by minimizing the trace of obtained upper bound of the state estimation error covariance at each step; 3 ) the desired performance analysis is conducted and a sufficient criterion is given to verify the exponential boundedness of the estimation error; and 4) the proposed estimation algorithm possesses the recursive form that is applicable for online computations.

Notations. The notations used throughout this paper are fairly standard except specifically stated. $\mathbb{R}^{n}$ stands for the $n$-dimensional Euclidean space. " $X^{T}$ " represents the transposition of matrix $X . X>0(X \geq 0)$ denotes that $X$ is a real symmetric and positive definite (positive semidefinite) matrix. $\mathbb{E}\{y\}$ stands for the mathematical expectation of $y \cdot \operatorname{diag}\left\{P_{1}, P_{2}, \cdots, P_{n}\right\}$ is a block-diagonal matrix with $P_{1}, P_{2} \cdots, P_{n}$ on the diagonal. $\operatorname{tr}(X)$ represents the trace of matrix $X . I$ and 0 denote the identity matrix and zero matrix with proper dimensions. $\otimes$ stands for the Kronecker product. Matrices, if their dimensions are not clearly stated, are assumed to be compatible for algebraic operations.

\section{Problem Formulation}

In this paper, we consider the following class of discrete time-varying stochastic complex networks consisting of $N$ coupled nodes:

$$
\begin{aligned}
x_{i, k+1} & =A_{i, k} x_{i, k}+\sum_{j=1}^{N} \omega_{i j} \Gamma x_{j, k}+B_{i, k} \varpi_{i, k}, \\
y_{i, k} & =C_{i, k} x_{i, k}+\nu_{i, k},
\end{aligned}
$$

where $x_{i, k} \in \mathbb{R}^{n}$ denotes the state of the $i$-th node $(i=$ $1,2, \cdots, N)$ with the initial state $x_{i, 0}, y_{i, k} \in \mathbb{R}^{m}$ stands for the measurement output from the $i$-th node, $\varpi_{i, k}$ and $\nu_{i, k}$ are zero-mean noises with covariances $Q_{i, k}>0$ and $R_{i, k}>0$, respectively. $A_{i, k}, B_{i, k}$ and $C_{i, k}$ are bounded and known matrices with appropriate dimensions. For the considered complex network, the coupling configuration matrix is denoted as $W=\left(\omega_{i j}\right)_{N \times N}$ with elements $\omega_{i j} \geq 0(i \neq j)$ but not all zero.

In (1), $\Gamma=\operatorname{diag}\left\{\gamma_{1}, \gamma_{2}, \cdots, \gamma_{N}\right\}$ is the inner coupling matrix, where $\gamma_{j} \neq 0$ represents the linking with the $j$-th state variable. Here, we consider the case that the coupling strength $\gamma_{i}(i=1,2, \cdots, N)$ is unknown but belongs to a certain interval $\left[\underline{\gamma}_{i}, \bar{\gamma}_{i}\right]$, where $\underline{\gamma}_{i}$ and $\bar{\gamma}_{i}$ are known scalars with $\underline{\gamma}_{i}<\bar{\gamma}_{i}$. Setting

$$
\bar{\Gamma}=\operatorname{diag}\left\{\frac{\underline{\gamma}_{1}+\bar{\gamma}_{1}}{2}, \frac{\underline{\gamma}_{2}+\bar{\gamma}_{2}}{2}, \cdots, \frac{\underline{\gamma}_{N}+\bar{\gamma}_{N}}{2}\right\},
$$




$$
\vec{\Gamma}=\operatorname{diag}\left\{\frac{\bar{\gamma}_{1}-\underline{\gamma}_{1}}{2}, \frac{\bar{\gamma}_{2}-\underline{\gamma}_{2}}{2}, \cdots, \frac{\bar{\gamma}_{N}-\underline{\gamma}_{N}}{2}\right\}
$$

then $\Gamma$ can be rewritten as $\Gamma=\bar{\Gamma}+\tilde{\Gamma}$ with $\tilde{\Gamma} \in[-\vec{\Gamma}, \vec{\Gamma}]$. Subsequently, the matrix $\Gamma$ can be further described by:

$$
\Gamma=\bar{\Gamma}+\mathcal{F} \vec{\Gamma}, \quad \mathcal{F} \mathcal{F}^{T}=\mathcal{F}^{T} \mathcal{F} \leq I,
$$

with $\mathcal{F}=\tilde{\Gamma} \vec{\Gamma}^{-1}$. Throughout the paper, suppose that the noises $\varpi_{i, k}$ and $\nu_{i, k}$ are mutually independent in $k$ and $i$.

Remark 1: In the time-varying complex networks (1)-(2), the first term in (1) represents its evolution behaviour of node $i$, the second term stands for the evolution effects induced by its adjacent nodes, and the third one reflects the process noise. In particular, the second term includes two aspects, i.e., the outer coupling parameters $\omega_{i j}$ reflect the connection from nodes $j$ to $i(j \neq i)$ at the time step $k$ if $\omega_{i j} \neq 0$, and the parameters $\gamma_{i}$ $(i=1,2, \cdots, N)$ depict the inner coupling strengthen of the system states. It should be noted that the coupling strengthens between the state elements are different when $\gamma_{i} \neq \gamma_{j}$, which can provide more flexibility on the modelling of complex networks. Besides, the measurement outputs are described by (2). Overall, the addressed complex networks model can be seen as a basic one in the existing literature, which can be used to characterize a variety of practical systems. For example, in the multiple mobile robotics applications (e.g. the formation problem), the dynamics trajectory of each robot depends on its state (e.g. position, velocity). Meanwhile, there is a need to integrate the states of other robots in order to achieve the formation, thus the formation of the multiple mobile robots can be modelled by (1). Consequently, efficient estimation approach and thorough performance analysis are required to handle the variance-constrained state estimation problem for addressed time-varying complex networks (1)-(2).

During the signal transmission, the signal quantization phenomenon is likely to occur since the communication capacity of the network channels is limited. Here, the mapping of the quantization process is described by

$$
q\left(y_{i, k}\right)=\left[\begin{array}{llll}
q_{1}\left(y_{i, k}^{(1)}\right) & q_{2}\left(y_{i, k}^{(2)}\right) & \ldots & q_{m}\left(y_{i, k}^{(m)}\right)
\end{array}\right]^{T} .
$$

For each $q_{j}(\cdot)$, the set of quantization levels is given by

$$
\begin{array}{r}
\mathscr{U}_{j}=\left\{ \pm u_{l}^{(j)}, u_{l}^{(j)}=\left(\chi^{(j)}\right)^{l} u_{0}^{(j)}, l=0, \pm 1, \pm 2, \ldots\right\} \\
\cup\{0\}, 0<\chi^{(j)}<1, u_{0}^{(j)}>0,
\end{array}
$$

where $\chi^{(j)}(j=1,2, \ldots, m)$ denotes the quantization density, and $u_{l}^{(j)}$ means the quantization level. Next, as in [10], the following logarithmic quantizer is adopted

$$
q_{j}\left(y_{i, k}^{(j)}\right)=\left\{\begin{array}{cc}
u_{l}^{(j)}, & \frac{1}{1+\delta_{j}} u_{l}^{(j)}<y_{i, k}^{(j)} \leq \frac{1}{1-\delta_{j}} u_{l}^{(j)} \\
0, & y_{i, k}^{(j)}=0 \\
-q_{j}\left(-y_{i, k}^{(j)}\right), & y_{i, k}^{(j)}<0
\end{array}\right.
$$

where $\delta_{j}=\frac{1-\chi^{(j)}}{1+\chi^{(j)}}$. From the above definition, we have $q_{j}\left(y_{i, k}^{(j)}\right)=\left(1+\Delta_{k}^{(j)}\right) y_{i, k}^{(j)}$ with $\left|\Delta_{k}^{(j)}\right| \leq \delta_{j}$.
Setting $\Delta_{k}=\operatorname{diag}\left\{\Delta_{k}^{(1)}, \Delta_{k}^{(2)}, \ldots, \Delta_{k}^{(m)}\right\}$, the measurements transmitted to the state estimator can be rewritten as

$$
\tilde{y}_{i, k}=q\left(y_{i, k}\right)=\left(I+\Delta_{k}\right) C_{i, k} x_{i, k}+\left(I+\Delta_{k}\right) \nu_{k} .
$$

Letting $\Lambda=\operatorname{diag}\left\{\delta_{1}, \delta_{2}, \cdots, \delta_{m}\right\}$ and $\mathscr{F}_{k}=\Delta_{k} \Lambda^{-1}$, we have $\Delta_{k}=\mathscr{F}_{k} \Lambda$ with $\mathscr{F}_{k}$ satisfying $\mathscr{F}_{k} \mathscr{F}_{k}^{T}=\mathscr{F}_{k}^{T} \mathscr{F}_{k} \leq I$.

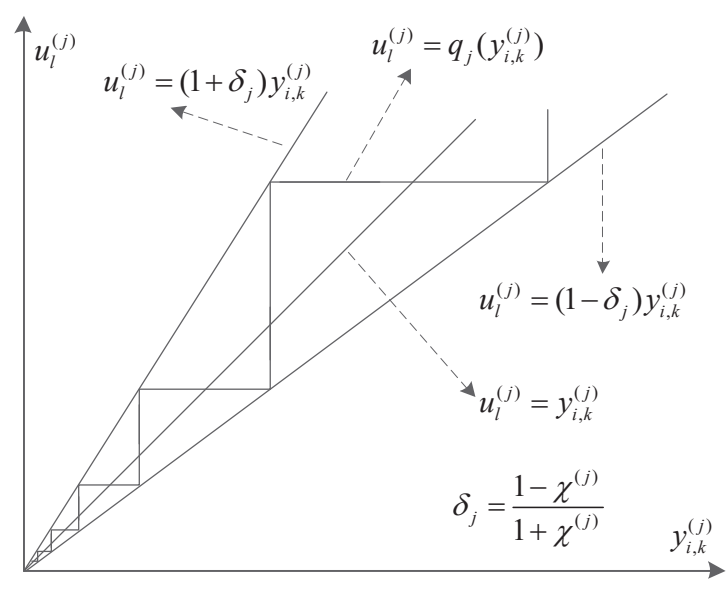

Fig. 1. The logarithmic quantizer.

Remark 2: Together with (4) and Fig. 1 as in [10], it is observed that the logarithmic quantizer can be bounded by a sector and the sector bound is expressed by the quantization density $\chi^{(j)}(j=1,2, \ldots, m)$ (or the parameter $\delta_{j}$ ).

For node $i$, the following time-varying state estimator is designed:

$$
\begin{aligned}
\hat{x}_{i, k+1 \mid k} & =A_{i, k} \hat{x}_{i, k \mid k}+\sum_{j=1}^{N} \omega_{i j} \bar{\Gamma} \hat{x}_{j, k \mid k} \\
\hat{x}_{i, k+1 \mid k+1} & =\hat{x}_{i, k+1 \mid k}+K_{i, k+1}\left(\tilde{y}_{i, k+1}-C_{i, k+1} \hat{x}_{i, k+1 \mid k}\right)
\end{aligned}
$$

where $\hat{x}_{i, k+1 \mid k}$ denotes the one-step prediction at time $k, \hat{x}_{i, k \mid k}$ $(i=1,2, \ldots, N)$ represents the state estimate of $x_{i, k}$ at time step $k$ with $\hat{x}_{i, 0 \mid 0}, \tilde{y}_{i, k+1}$ is the actual input to the estimator on node $i$, and $K_{i, k+1}$ is the desired estimator parameter matrix to be determined.

For node $i$, let $\tilde{x}_{i, k+1 \mid k}=x_{i, k+1}-\hat{x}_{i, k+1 \mid k}$ represent the one-step prediction error and $\tilde{x}_{i, k+1 \mid k+1}=x_{i, k+1}-\hat{x}_{i, k+1 \mid k+1}$ denote the estimation error. Then, it follows from (1) and (6) that

$$
\begin{aligned}
\tilde{x}_{i, k+1 \mid k}= & A_{i, k} \tilde{x}_{i, k \mid k}+\sum_{j=1}^{N} \omega_{i j} \bar{\Gamma}\left(x_{j, k}-\hat{x}_{j, k \mid k}\right) \\
& +\sum_{j=1}^{N} \omega_{i j} \tilde{\Gamma} x_{j, k}+B_{i, k} \varpi_{i, k} .
\end{aligned}
$$

Similarly, together with (5) and (7), we have

$$
\begin{aligned}
\tilde{x}_{i, k+1 \mid k+1}= & \left(I-K_{i, k+1} C_{i, k+1}\right) \tilde{x}_{i, k+1 \mid k} \\
& -K_{i, k+1} \Delta_{k+1} C_{i, k+1} x_{i, k+1} \\
& -K_{i, k+1}\left(I+\Delta_{k+1}\right) \nu_{i, k+1} .
\end{aligned}
$$


To proceed, we set

$$
\begin{aligned}
& \mathbb{I}=\operatorname{diag}\{\underbrace{I, I, \ldots, I}_{N}\}, \\
& \tilde{\Delta}_{k}=\operatorname{diag}\{\underbrace{\Delta_{k}, \Delta_{k}, \ldots, \Delta_{k}}_{N}\} \text {, } \\
& A_{k}=\operatorname{diag}\left\{A_{1, k}, A_{2, k}, \ldots, A_{N, k}\right\} \text {, } \\
& K_{k}=\operatorname{diag}\left\{K_{1, k}, K_{2, k}, \ldots, K_{N, k}\right\} \text {, } \\
& B_{k}=\operatorname{diag}\left\{B_{1, k}, B_{2, k}, \ldots, B_{N, k}\right\} \text {, } \\
& C_{k}=\operatorname{diag}\left\{C_{1, k}, C_{2, k}, \ldots, C_{N, k}\right\} \text {, } \\
& x_{k}=\left[\begin{array}{llll}
x_{1, k}^{T} & x_{2, k}^{T} & \ldots & x_{N, k}^{T}
\end{array}\right]^{T}, \\
& \varpi_{k}=\left[\begin{array}{llll}
\varpi_{1, k}^{T} & \varpi_{2, k}^{T} & \cdots & \varpi_{N, k}^{T}
\end{array}\right]^{T}, \\
& \nu_{k}=\left[\begin{array}{llll}
\nu_{1, k}^{T} & \nu_{2, k}^{T} & \cdots & \nu_{N, k}^{T}
\end{array}\right]^{T}, \\
& \hat{x}_{k+1 \mid k}=\left[\begin{array}{llll}
\hat{x}_{1, k+1 \mid k}^{T} & \hat{x}_{2, k+1 \mid k}^{T} & \cdots & \hat{x}_{N, k+1 \mid k}^{T}
\end{array}\right]^{T}, \\
& \hat{x}_{k+1 \mid k+1}=\left[\begin{array}{llll}
\hat{x}_{1, k+1 \mid k+1}^{T} & \hat{x}_{2, k+1 \mid k+1}^{T} & \ldots & \hat{x}_{N, k+1 \mid k+1}^{T}
\end{array}\right]^{T}, \\
& \tilde{x}_{k+1 \mid k}=\left[\begin{array}{llll}
\tilde{x}_{1, k+1 \mid k}^{T} & \tilde{x}_{2, k+1 \mid k}^{T} & \ldots & \tilde{x}_{N, k+1 \mid k}^{T}
\end{array}\right]^{T}, \\
& \tilde{x}_{k+1 \mid k+1}=\left[\begin{array}{llll}
\tilde{x}_{1, k+1 \mid k+1}^{T} & \tilde{x}_{2, k+1 \mid k+1}^{T} & \ldots & \tilde{x}_{N, k+1 \mid k+1}^{T}
\end{array}\right]^{T} .
\end{aligned}
$$

Then, based on the above notations and using the Kronecker product, we can rewrite the one-step prediction error $\tilde{x}_{k+1 \mid k}$ and the estimation error $\tilde{x}_{k+1 \mid k+1}$ as

$$
\begin{aligned}
\tilde{x}_{k+1 \mid k}= & \left(A_{k}+W \otimes \bar{\Gamma}\right) \tilde{x}_{k \mid k}+(W \otimes \tilde{\Gamma}) x_{k} \\
& +B_{k} \varpi_{k}, \\
\tilde{x}_{k+1 \mid k+1}= & \Pi_{k+1} \tilde{x}_{k+1 \mid k}-K_{k+1} \tilde{\Delta}_{k+1} C_{k+1} x_{k+1} \\
& -K_{k+1}\left(\mathbb{I}+\tilde{\Delta}_{k+1}\right) \nu_{k+1},
\end{aligned}
$$

where $\Pi_{k+1}=\mathbb{I}-K_{k+1} C_{k+1}$.

Now, let $P_{k+1 \mid k+1}=\mathbb{E}\left\{\tilde{x}_{k+1 \mid k+1} \tilde{x}_{k+1 \mid k+1}^{T}\right\}$ be the estimation error covariance at the $k+1$-th time step. Then, the objectives of the paper can be given as follows.

(i) We aim to design a time-varying estimator of form (6)(7) such that an upper bound $\Omega_{k+1 \mid k+1}$ of the estimation error covariance $P_{k+1 \mid k+1}$ is guaranteed, i.e.,

$$
P_{k+1 \mid k+1} \leq \Omega_{k+1 \mid k+1} .
$$

In addition, the trace of the obtained upper bound $\Omega_{k+1 \mid k+1}$ is minimized by determining the estimator gain matrix at each time step.

(ii) A sufficient condition is given to guarantee the exponential mean-square boundedness of the estimation error.

Remark 3: Note that the time-varying complex networks with Gaussian white noises are considered in this paper. Hence, the variance-constrained estimation method is proposed, which can deal with the effects from the Gaussian white noises, uncertain inner coupling and quantization errors in a unified framework. In addition, the newly proposed varianceconstrained estimation scheme can provide satisfactory estimation performance at the worst situation. As will be shown later, with the designed state estimator (6)-(7), the estimation algorithm to be presented has the following advantages: (a) the state estimator structure is simple and easy-to-implement especially in the time-varying case; (b) the state estimations from adjacent nodes and the innovation information are both employed when constructing the estimator; (c) the impacts induced by the uncertain inner coupling, quantization errors and different noises are explicitly examined in the proposed algorithm; and (d) the new variance-constrained estimation scheme is of a recursive feature applicable for online computations. On the other hand, in (12), the term $P_{k+1 \mid k+1}$ represents the covariance of the estimation error covariance, which can characterize the estimation performance of the proposed method. According to (12), $P_{k+1 \mid k+1}-\Omega_{k+1 \mid k+1} \leq 0$ means that $P_{k+1 \mid k+1}-\Omega_{k+1 \mid k+1}$ is negative semi-definite matrix. In this case, this paper provides an alternative method to evaluate the estimation performance of the developed method to an extent.

\section{MAin Results}

To proceed, the following lemmas are introduced which will be used in the design of the variance-constrained estimation algorithm.

Lemma 1: [12] For real vectors $a$ and $b$ with appropriate dimensions and a scalar $\varepsilon>0$, we have

$$
a b^{T}+b a^{T} \leq \varepsilon a a^{T}+\varepsilon^{-1} b b^{T} .
$$

Lemma 2: [35] For given matrices $A, H, E$ and $F$ with $F F^{T} \leq I$, let $\gamma^{-1} I-E X E^{T}>0$ with $X>0$ and $\gamma>0$, the following inequality

$$
\begin{aligned}
& (A+H F E) X(A+H F E)^{T} \\
\leq & A\left(X^{-1}-\gamma E^{T} E\right)^{-1} A^{T}+\gamma^{-1} H H^{T}
\end{aligned}
$$

holds.

Lemma 3: [8] For real matrices $M, X, N$ and $P$, we have the following properties:

$$
\begin{aligned}
& \frac{\partial \operatorname{tr}(M X N)}{\partial X}=M^{T} N^{T}, \quad \frac{\partial \operatorname{tr}\left(M X^{T} N\right)}{\partial X}=N M, \\
& \frac{\partial \operatorname{tr}\left[(M X N) P(M X N)^{T}\right]}{\partial X}=2 M^{T} M X N P N^{T} .
\end{aligned}
$$

Based on the definitions of the one-step prediction error and the state estimation error, the following two lemmas can be established in which both the one-step prediction error covariance and the estimation error covariance are calculated, respectively.

Lemma 4: The recursion of the one-step prediction error covariance $P_{k+1 \mid k}=\mathbb{E}\left\{\tilde{x}_{k+1 \mid k} \tilde{x}_{k+1 \mid k}^{T}\right\}$ can be calculated by:

$$
\begin{aligned}
P_{k+1 \mid k}= & \left(A_{k}+W \otimes \bar{\Gamma}\right) P_{k \mid k}\left(A_{k}+W \otimes \bar{\Gamma}\right)^{T} \\
& +\left(A_{k}+W \otimes \bar{\Gamma}\right) \mathbb{E}\left\{\tilde{x}_{k \mid k} x_{k}^{T}\right\}(W \otimes \tilde{\Gamma})^{T} \\
& +(W \otimes \tilde{\Gamma}) \mathbb{E}\left\{x_{k} \tilde{x}_{k \mid k}^{T}\right\}\left(A_{k}+W \otimes \bar{\Gamma}\right)^{T} \\
& +(W \otimes \tilde{\Gamma}) \mathbb{E}\left\{x_{k} x_{k}^{T}\right\}(W \otimes \tilde{\Gamma})^{T} \\
& +B_{k} Q_{k} B_{k}^{T}
\end{aligned}
$$

where

$$
Q_{k}=\operatorname{diag}\left\{Q_{1, k}, Q_{2, k}, \cdots, Q_{N, k}\right\} .
$$


Proof: Based on the definition of $P_{k+1 \mid k}$ and according to $(10)$, one has

$$
\begin{aligned}
P_{k+1 \mid k}= & \mathbb{E}\left\{\tilde{x}_{k+1 \mid k} \tilde{x}_{k+1 \mid k}^{T}\right\} \\
= & \left(A_{k}+W \otimes \bar{\Gamma}\right) P_{k \mid k}\left(A_{k}+W \otimes \bar{\Gamma}\right)^{T} \\
& +\left(A_{k}+W \otimes \bar{\Gamma}\right) \mathbb{E}\left\{\tilde{x}_{k \mid k} x_{k}^{T}\right\}(W \otimes \tilde{\Gamma})^{T} \\
& +(W \otimes \tilde{\Gamma}) \mathbb{E}\left\{x_{k} \tilde{x}_{k \mid k}^{T}\right\}\left(A_{k}+W \otimes \bar{\Gamma}\right)^{T} \\
& +\left(A_{k}+W \otimes \tilde{\Gamma}\right) \mathbb{E}\left\{\tilde{x}_{k \mid k} \varpi_{k}^{T}\right\} B_{k}^{T} \\
& +B_{k} \mathbb{E}\left\{\varpi_{k} \tilde{x}_{k \mid k}^{T}\right\}\left(A_{k}+W \otimes \bar{\Gamma}\right)^{T} \\
& +(W \otimes \tilde{\Gamma}) \mathbb{E}\left\{x_{k} x_{k}^{T}\right\}(W \otimes \tilde{\Gamma})^{T} \\
& +(W \otimes \tilde{\Gamma}) \mathbb{E}\left\{x_{k} \varpi_{k}^{T}\right\} B_{k}^{T} \\
& +B_{k} \mathbb{E}\left\{\varpi_{k} x_{k}^{T}\right\}(W \otimes \tilde{\Gamma})^{T} \\
& +B_{k} Q_{k} B_{k}^{T} \\
= & \left(A_{k}+W \otimes \bar{\Gamma}\right) P_{k \mid k}\left(A_{k}+W \otimes \bar{\Gamma}\right)^{T} \\
& +\left(A_{k}+W \otimes \bar{\Gamma}\right) \mathbb{E}\left\{\tilde{x}_{k \mid k} x_{k}^{T}\right\}(W \otimes \tilde{\Gamma})^{T} \\
& +(W \otimes \tilde{\Gamma}) \mathbb{E}\left\{x_{k} \tilde{x}_{k \mid k}^{T}\right\}\left(A_{k}+W \otimes \bar{\Gamma}\right)^{T} \\
& +(W \otimes \tilde{\Gamma}) \mathbb{E}\left\{x_{k} x_{k}^{T}\right\}(W \otimes \tilde{\Gamma})^{T} \\
& +B_{k} Q_{k} B_{k}^{T},
\end{aligned}
$$

where $Q_{k}$ is defined as in (16). When deriving (17), we have used the fact that $\varpi_{k}$ is zero-mean. Then, the proof of this theorem is complete.

Lemma 5: The evolution of the estimation error covariance $P_{k+1 \mid k+1}$ can be calculated by:

$$
\begin{aligned}
& P_{k+1 \mid k+1} \\
= & \Pi_{k+1} P_{k+1 \mid k} \Pi_{k+1}^{T} \\
& -\Pi_{k+1} \mathbb{E}\left\{\tilde{x}_{k+1 \mid k} x_{k+1}^{T}\right\} C_{k+1}^{T} \tilde{\Delta}_{k+1}^{T} K_{k+1}^{T} \\
& -K_{k+1} \tilde{\Delta}_{k+1} C_{k+1} \mathbb{E}\left\{x_{k+1} \tilde{x}_{k+1 \mid k}^{T}\right\} \Pi_{k+1}^{T} \\
& +K_{k+1} \tilde{\Delta}_{k+1} C_{k+1} \mathbb{E}\left\{x_{k+1} x_{k+1}^{T}\right\} C_{k+1}^{T} \tilde{\Delta}_{k+1}^{T} K_{k+1}^{T} \\
& +K_{k+1}\left(\mathbb{I}+\tilde{\Delta}_{k+1}\right) R_{k+1}\left(\mathbb{I}+\tilde{\Delta}_{k+1}\right)^{T} K_{k+1}^{T},
\end{aligned}
$$

where

$$
R_{k+1}=\operatorname{diag}\left\{R_{1, k+1}, R_{2, k+1}, \cdots, R_{N, k+1}\right\} .
$$

Proof: It follows from (11) that

$$
\begin{aligned}
& P_{k+1 \mid k+1} \\
= & \mathbb{E}\left\{\tilde{x}_{k+1 \mid k+1} \tilde{x}_{k+1 \mid k+1}^{T}\right\} \\
= & \Pi_{k+1} P_{k+1 \mid k} \Pi_{k+1}^{T} \\
& -\Pi_{k+1} \mathbb{E}\left\{\tilde{x}_{k+1 \mid k} x_{k+1}^{T}\right\} C_{k+1}^{T} \tilde{\Delta}_{k+1}^{T} K_{k+1}^{T} \\
& -K_{k+1} \tilde{\Delta}_{k+1} C_{k+1} \mathbb{E}\left\{x_{k+1} \tilde{x}_{k+1 \mid k}^{T}\right\} \Pi_{k+1}^{T} \\
& +K_{k+1} \tilde{\Delta}_{k+1} C_{k+1} \mathbb{E}\left\{x_{k+1} x_{k+1}^{T}\right\} C_{k+1}^{T} \tilde{\Delta}_{k+1}^{T} K_{k+1}^{T} \\
& +K_{k+1}\left(\mathbb{I}+\tilde{\Delta}_{k+1}\right) \mathbb{E}\left\{\nu_{k+1} \nu_{k+1}^{T}\right\}\left(\mathbb{I}+\tilde{\Delta}_{k+1}\right)^{T} K_{k+1}^{T} \\
& -\mathscr{A}_{k+1}-\mathscr{A}_{k+1}^{T}+\mathscr{B}_{k+1}+\mathscr{B}_{k+1}^{T}
\end{aligned}
$$

where

$$
\begin{aligned}
\mathscr{A}_{k+1}= & \Pi_{k+1} \mathbb{E}\left\{\tilde{x}_{k+1 \mid k} \nu_{k+1}^{T}\right\}\left(\mathbb{I}+\tilde{\Delta}_{k+1}\right)^{T} K_{k+1}^{T}, \\
\mathscr{B}_{k+1}= & K_{k+1} \tilde{\Delta}_{k+1} C_{k+1} \mathbb{E}\left\{x_{k+1} \nu_{k+1}^{T}\right\} \\
& \times\left(\mathbb{I}+\tilde{\Delta}_{k+1}\right)^{T} K_{k+1}^{T} .
\end{aligned}
$$

Subsequently, it is easy to verify that both $\mathscr{A}_{k+1}$ and $\mathscr{B}_{k+1}$ are equal to zero. Then, we can conclude that (18) holds and this ends the proof of this theorem.

It is worth noting that the obtained estimation error covariance contains some unknown terms, and it is therefore difficult to design the estimator parameter matrix that minimizes the trace of the resulting estimation error covariance. In what follows, an alternative method is employed, with which an upper bound of the estimation error covariance is firstly derived by using the matrix analysis technique, and then the trace of the obtained upper bound is minimized by properly choosing the estimator parameter matrix at every time step. Moreover, additional assumptions can be made on the system parameters, the parameters of uncertain inner coupling and quantization errors, and the covariance of the process noise, thereby the corresponding boundedness analysis can be proposed as shown later.

Theorem 1: For given positive scalars $\epsilon_{1, k}, \epsilon_{2, k}, \epsilon_{3, k+1}$, $\epsilon_{4, k+1}$ and $\varepsilon_{k+1}$, if the following two recursive matrix equations

$$
\begin{aligned}
\Omega_{k+1 \mid k}= & \left(1+\epsilon_{1, k}\right)\left(A_{k}+W \otimes \bar{\Gamma}\right) \Omega_{k \mid k}\left(A_{k}+W \otimes \bar{\Gamma}\right)^{T} \\
& +\left(1+\epsilon_{1, k}^{-1}\right) \operatorname{tr}\left(\check { \Gamma } \left[\left(1+\epsilon_{2, k}\right) \Omega_{k \mid k}+\left(1+\epsilon_{2, k}^{-1}\right)\right.\right. \\
& \left.\left.\times \hat{x}_{k \mid k} \hat{x}_{k \mid k}^{T}\right] \check{\Gamma}^{T}\right) \check{W} \check{W}^{T}+B_{k} Q_{k} B_{k}^{T}
\end{aligned}
$$

and

$$
\begin{aligned}
& \Omega_{k+1 \mid k+1} \\
= & \left(1+\epsilon_{3, k+1}\right) \Pi_{k+1} \Omega_{k+1 \mid k} \Pi_{k+1}^{T} \\
& +K_{k+1}\left\{( 1 + \epsilon _ { 3 , k + 1 } ^ { - 1 } ) \operatorname { t r } \left(\tilde { \Lambda } C _ { k + 1 } \left[\left(1+\epsilon_{4, k+1}\right) \Omega_{k+1 \mid k}\right.\right.\right. \\
& \left.\left.+\left(1+\epsilon_{4, k+1}^{-1}\right) \hat{x}_{k+1 \mid k} \hat{x}_{k+1 \mid k}^{T}\right] C_{k+1}^{T} \tilde{\Lambda}^{T}\right) \mathbb{I} \\
& \left.+\left(R_{k+1}^{-1}-\varepsilon_{k+1} \tilde{\Lambda}^{T} \tilde{\Lambda}\right)^{-1}+\varepsilon_{k+1}^{-1} \mathbb{I}\right\} K_{k+1}^{T}
\end{aligned}
$$

under $\Omega_{0 \mid 0}=P_{0 \mid 0}$ and the following constraint

$$
\varepsilon_{k+1}^{-1} \mathbb{I}-\tilde{\Lambda} R_{k+1} \tilde{\Lambda}^{T}>0,
$$

have symmetric positive definite solutions, then it can be shown that $\Omega_{k+1 \mid k+1}$ is an upper bound of $P_{k+1 \mid k+1}$, that is,

$$
P_{k+1 \mid k+1} \leq \Omega_{k+1 \mid k+1},
$$

where

$$
\begin{aligned}
& \check{W}=W \otimes I, \\
& \tilde{\Lambda}=\operatorname{diag}\{\underbrace{\Lambda, \Lambda, \cdots, \Lambda}_{N}\}, \\
& \check{\Gamma}=\operatorname{diag}\{\underbrace{\vec{\Gamma}, \vec{\Gamma}, \cdots, \vec{\Gamma}}_{N}\}, \\
& \Psi_{1, i}=\left[\begin{array}{llll}
\underbrace{\begin{array}{llll}
0 & \cdots & \cdots & 0
\end{array}}_{i-1} I_{n \times n} & \underbrace{\begin{array}{llll}
0 & 0 & \cdots & 0
\end{array}}_{N-i}
\end{array}\right], \\
& \Psi_{2, i}=\left[\begin{array}{llllll}
\underbrace{\begin{array}{lllll}
0 & 0 & \cdots & 0
\end{array}}_{i-1} & I_{m \times m} & \underbrace{\begin{array}{lllll}
0 & 0 & \cdots & 0
\end{array}}_{N-i}
\end{array}\right] .
\end{aligned}
$$

Moreover, if we adopt the following estimator parameter

$$
K_{k+1}=\operatorname{diag}\left\{K_{1, k+1}, K_{2, k+1}, \ldots, K_{N, k+1}\right\},
$$


with

$$
\begin{aligned}
K_{i, k+1}= & \left(1+\epsilon_{3, k+1}\right) \Psi_{1, i} \Omega_{k+1 \mid k} C_{k+1}^{T} \Psi_{2, i}^{T} \\
& \times\left(\Psi_{2, i} \Xi_{k+1} \Psi_{2, i}^{T}\right)^{-1} \\
\Xi_{k+1}= & \left(1+\epsilon_{3, k+1}\right) C_{k+1} \Omega_{k+1 \mid k} C_{k+1}^{T} \\
& +\left(R_{k+1}^{-1}-\varepsilon_{k+1} \tilde{\Lambda}^{T} \tilde{\Lambda}\right)^{-1}+\varepsilon_{k+1}^{-1} \mathbb{I} \\
& +\left(1+\epsilon_{3, k+1}^{-1}\right) \operatorname{tr}\left(\tilde { \Lambda } C _ { k + 1 } \left[\left(1+\epsilon_{4, k+1}\right) \Omega_{k+1 \mid k}\right.\right. \\
& \left.\left.+\left(1+\epsilon_{4, k+1}^{-1}\right) \hat{x}_{k+1 \mid k} \hat{x}_{k+1 \mid k}^{T}\right] C_{k+1}^{T} \tilde{\Lambda}^{T}\right) \mathbb{I},
\end{aligned}
$$

then the trace of the obtained upper bound $\Omega_{k+1 \mid k+1}$ can be minimized at each sampling instant.

Proof: By using the stochastic analysis technique and the mathematical induction approach, we take three steps to verify the statement in this theorem.

Firstly, let us deal with the uncertain terms of the right-hand side of (15). Based on Lemma 1, one has

$$
\begin{aligned}
& \left(A_{k}+W \otimes \bar{\Gamma}\right) \mathbb{E}\left\{\tilde{x}_{k \mid k} x_{k}^{T}\right\}(W \otimes \tilde{\Gamma})^{T} \\
& +(W \otimes \tilde{\Gamma}) \mathbb{E}\left\{x_{k} \tilde{x}_{k \mid k}^{T}\right\}\left(A_{k}+W \otimes \bar{\Gamma}\right)^{T} \\
\leq & \epsilon_{1, k}\left(A_{k}+W \otimes \bar{\Gamma}\right) P_{k \mid k}\left(A_{k}+W \otimes \bar{\Gamma}\right)^{T} \\
+ & \epsilon_{1, k}^{-1}(W \otimes \tilde{\Gamma}) \mathbb{E}\left\{x_{k} x_{k}^{T}\right\}(W \otimes \tilde{\Gamma})^{T}
\end{aligned}
$$

where $\epsilon_{1, k}$ is a positive scalar. In view of the definition of $\tilde{x}_{k \mid k}$ and by employing Lemma 1 again, we have

$$
\begin{aligned}
& \mathbb{E}\left\{x_{k} x_{k}^{T}\right\} \\
\leq & \left(1+\epsilon_{2, k}\right) P_{k \mid k}+\left(1+\epsilon_{2, k}^{-1}\right) \hat{x}_{k \mid k} \hat{x}_{k \mid k}^{T} \\
:= & \overline{\mathcal{S}}_{k},
\end{aligned}
$$

where $\epsilon_{2, k}$ is a positive scalar. In view of (15), (28) and (29), we obtain

$$
\begin{aligned}
& P_{k+1 \mid k} \\
\leq & \left(1+\epsilon_{1, k}\right)\left(A_{k}+W \otimes \bar{\Gamma}\right) P_{k \mid k}\left(A_{k}+W \otimes \bar{\Gamma}\right)^{T} \\
& +\left(1+\epsilon_{1, k}^{-1}\right)(W \otimes \tilde{\Gamma}) \overline{\mathcal{S}}_{k}(W \otimes \tilde{\Gamma})^{T}+B_{k} Q_{k} B_{k}^{T} .
\end{aligned}
$$

Subsequently, note that $W \otimes \tilde{\Gamma}=\check{W} \check{\mathcal{F}} \check{\Gamma}$, where $\check{W}$ and $\check{\Gamma}$ are defined as in (25), and $\check{\mathcal{F}}=\operatorname{diag}\{\underbrace{\mathcal{F}, \mathcal{F}, \cdots, \mathcal{F}}_{N}\}$. Then, one has

$$
\begin{aligned}
& \left(1+\epsilon_{1, k}^{-1}\right)(W \otimes \tilde{\Gamma}) \overline{\mathcal{S}}_{k}(W \otimes \tilde{\Gamma})^{T} \\
= & \left(1+\epsilon_{1, k}^{-1}\right) \check{W} \check{\mathcal{F}} \check{\Gamma} \overline{\mathcal{S}}_{k} \check{\Gamma}^{T} \check{\mathcal{F}}^{T} \check{W}^{T} \\
\leq & \left(1+\epsilon_{1, k}^{-1}\right) \operatorname{tr}\left(\check{\Gamma} \overline{\mathcal{S}}_{k} \check{\Gamma}^{T}\right) \check{W} \check{W}^{T} .
\end{aligned}
$$

Therefore, from (30) and (31), we arrive at

$$
\begin{aligned}
& P_{k+1 \mid k} \\
\leq & \left(1+\epsilon_{1, k}\right)\left(A_{k}+W \otimes \bar{\Gamma}\right) P_{k \mid k}\left(A_{k}+W \otimes \bar{\Gamma}\right)^{T} \\
& +\left(1+\epsilon_{1, k}^{-1}\right) \operatorname{tr}\left(\check{\Gamma}_{\overline{\mathcal{S}}} \check{\Gamma}^{T}\right) \check{W} \check{W}^{T}+B_{k} Q_{k} B_{k}^{T} .
\end{aligned}
$$

Secondly, we shall to handle unknown terms of the righthand side of (18). Based on Lemma 1 again, we have

$$
\begin{aligned}
& -\Pi_{k+1} \mathbb{E}\left\{\tilde{x}_{k+1 \mid k} x_{k+1}^{T}\right\} C_{k+1}^{T} \tilde{\Delta}_{k+1}^{T} K_{k+1}^{T} \\
& -K_{k+1} \tilde{\Delta}_{k+1} C_{k+1} \mathbb{E}\left\{x_{k+1} \tilde{x}_{k+1 \mid k}^{T}\right\} \Pi_{k+1}^{T} \\
\leq & \epsilon_{3, k+1} \Pi_{k+1} P_{k+1 \mid k} \Pi_{k+1}^{T}+\epsilon_{3, k+1}^{-1} K_{k+1} \tilde{\Delta}_{k+1}
\end{aligned}
$$

$$
\times C_{k+1} \mathbb{E}\left\{x_{k+1} x_{k+1}^{T}\right\} C_{k+1}^{T} \tilde{\Delta}_{k+1}^{T} K_{k+1}^{T},
$$

where $\epsilon_{3, k+1}>0$ is a scalar. Substituting (33) into (18) yields

$$
\begin{aligned}
& P_{k+1 \mid k+1} \\
\leq & \left(1+\epsilon_{3, k+1}\right) \Pi_{k+1} P_{k+1 \mid k} \Pi_{k+1}^{T} \\
& +\left(1+\epsilon_{3, k+1}^{-1}\right) K_{k+1} \tilde{\Delta}_{k+1} C_{k+1} \mathbb{E}\left\{x_{k+1} x_{k+1}^{T}\right\} \\
& \times C_{k+1}^{T} \tilde{\Delta}_{k+1}^{T} K_{k+1}^{T}+K_{k+1}\left(\mathbb{I}+\tilde{\Delta}_{k+1}\right) \\
& \times R_{k+1}\left(\mathbb{I}+\tilde{\Delta}_{k+1}\right)^{T} K_{k+1}^{T} .
\end{aligned}
$$

Similarly, as in (29), we obtain

$$
\begin{aligned}
& \mathbb{E}\left\{x_{k+1} x_{k+1}^{T}\right\} \\
\leq & \left(1+\epsilon_{4, k+1}\right) P_{k+1 \mid k}+\left(1+\epsilon_{4, k+1}^{-1}\right) \hat{x}_{k+1 \mid k} \hat{x}_{k+1 \mid k}^{T} \\
:= & \overline{\mathcal{T}}_{k+1}
\end{aligned}
$$

where $\epsilon_{4, k+1}>0$ is a scalar. Then, it follows from (35) and $\tilde{\Delta}_{k+1}=\tilde{\mathscr{F}}_{k+1} \tilde{\Lambda}$ that

$$
\begin{aligned}
& K_{k+1} \tilde{\Delta}_{k+1} C_{k+1} \mathbb{E}\left\{x_{k+1} x_{k+1}^{T}\right\} C_{k+1}^{T} \tilde{\Delta}_{k+1}^{T} K_{k+1}^{T} \\
= & K_{k+1} \tilde{\mathscr{F}}_{k+1} \tilde{\Lambda} C_{k+1} \mathbb{E}\left\{x_{k+1} x_{k+1}^{T}\right\} C_{k+1}^{T} \tilde{\Lambda}^{T} \tilde{\mathscr{F}}_{k+1}^{T} K_{k+1}^{T} \\
\leq & K_{k+1} \tilde{\mathscr{F}}_{k+1} \tilde{\Lambda} C_{k+1} \overline{\mathcal{T}}_{k+1} C_{k+1}^{T} \tilde{\Lambda}^{T} \tilde{\mathscr{F}}_{k+1}^{T} K_{k+1}^{T} \\
\leq & \operatorname{tr}\left(\tilde{\Lambda} C_{k+1} \overline{\mathcal{T}}_{k+1} C_{k+1}^{T} \tilde{\Lambda}^{T}\right) K_{k+1} K_{k+1}^{T},
\end{aligned}
$$

where $\tilde{\mathscr{F}}_{k+1}=\operatorname{diag}\{\underbrace{\mathscr{F}_{k+1}, \mathscr{F}_{k+1}, \cdots, \mathscr{F}_{k+1}}_{N}\}$ and $\tilde{\Lambda}$ is defined in (25). Next, together with Lemma 2 and (23) that the last term of the right-hand side of (18) can be calculated

$$
\begin{aligned}
& K_{k+1}\left(\mathbb{I}+\tilde{\Delta}_{k+1}\right) R_{k+1}\left(\mathbb{I}+\tilde{\Delta}_{k+1}\right)^{T} K_{k+1}^{T} \\
= & K_{k+1}\left(\mathbb{I}+\tilde{\mathscr{F}}_{k+1} \tilde{\Lambda}\right) R_{k+1}\left(\mathbb{I}+\tilde{\mathscr{F}}_{k+1} \tilde{\Lambda}\right)^{T} K_{k+1}^{T} \\
\leq & K_{k+1}\left[\left(R_{k+1}^{-1}-\varepsilon_{k+1} \tilde{\Lambda}^{T} \tilde{\Lambda}\right)^{-1}+\varepsilon_{k+1}^{-1} \mathbb{I}\right] K_{k+1}^{T} .
\end{aligned}
$$

Subsequently, substituting (36) and (37) into (34) yields

$$
\begin{aligned}
& P_{k+1 \mid k+1} \\
\leq & \left(1+\epsilon_{3, k+1}\right) \Pi_{k+1} P_{k+1 \mid k} \Pi_{k+1}^{T} \\
& +\left(1+\epsilon_{3, k+1}^{-1}\right) \operatorname{tr}\left(\tilde{\Lambda} C_{k+1} \overline{\mathcal{T}}_{k+1} C_{k+1}^{T} \tilde{\Lambda}^{T}\right) K_{k+1} K_{k+1}^{T} \\
& +K_{k+1}\left[\left(R_{k+1}^{-1}-\varepsilon_{k+1} \tilde{\Lambda}^{T} \tilde{\Lambda}\right)^{-1}+\varepsilon_{k+1}^{-1} \mathbb{I}\right] K_{k+1}^{T} .
\end{aligned}
$$

Thirdly, we are in a position to show that $P_{k+1 \mid k+1} \leq$ $\Omega_{k+1 \mid k+1}$ in view of the mathematical induction approach. To prove the assertion, note the initial condition $P_{0 \mid 0}=\Omega_{0 \mid 0}$ and assume $P_{k \mid k} \leq \Omega_{k \mid k}$. Then, it follows from (21), (32), $\overline{\mathcal{S}}_{k}$ in (29) and $P_{k \mid k} \leq \Omega_{k \mid k}$ that

$$
P_{k+1 \mid k} \leq \Omega_{k+1 \mid k} .
$$

In the sequel, according to (22), (38), $\overline{\mathcal{T}}_{k+1}$ in (35) and $P_{k+1 \mid k} \leq \Omega_{k+1 \mid k}$, it is not difficult to testify that

$$
P_{k+1 \mid k+1} \leq \Omega_{k+1 \mid k+1} .
$$

Finally, we are ready to minimize the trace of the obtained upper bound $\Omega_{k+1 \mid k+1}$ by properly choosing the estimator parameter matrix $K_{i, k+1}$. Rewriting (22), we have

$$
\begin{aligned}
& \Omega_{k+1 \mid k+1} \\
= & \left(1+\epsilon_{3, k+1}\right) \Omega_{k+1 \mid k}-\left(1+\epsilon_{3, k+1}\right) \Omega_{k+1 \mid k} C_{k+1}^{T} K_{k+1}^{T} \\
& -\left(1+\epsilon_{3, k+1}\right) K_{k+1} C_{k+1} \Omega_{k+1 \mid k}+K_{k+1} \Xi_{k+1} K_{k+1}^{T},
\end{aligned}
$$


(40)

where $\Xi_{k+1}$ is defined as in (27). Notice that $K_{k+1}=$ $\sum_{i=1}^{N}\left(\Psi_{1, i}^{T} K_{i, k+1} \Psi_{2, i}\right)$, where $\Psi_{1, i}$ and $\Psi_{2, i}$ are defined as in (25). Then, it can be easily obtained that $\Psi_{1, j} \Psi_{1, i}^{T}=0$ for $i \neq j$. Thus, from the algebraic manipulations and the properties of trace, it follows that

$$
\begin{aligned}
& \operatorname{tr}\left[\left(\Psi_{1, i}^{T} K_{i, k+1} \Psi_{2, i}\right) \Xi_{k+1}\left(\Psi_{1, j}^{T} K_{j, k+1} \Psi_{2, j}\right)^{T}\right] \\
= & \operatorname{tr}\left[\Psi_{1, i}^{T} K_{i, k+1} \Psi_{2, i} \Xi_{k+1} \Psi_{2, j}^{T} K_{j, k+1}^{T} \Psi_{1, j}\right] \\
= & \operatorname{tr}\left[\Psi_{1, j} \Psi_{1, i}^{T} K_{i, k+1} \Psi_{2, i} \Xi_{k+1} \Psi_{2, j}^{T} K_{j, k+1}^{T}\right] \\
= & 0
\end{aligned}
$$

for $i \neq j$. Consequently, the trace of $\Omega_{k+1 \mid k+1}$ can be given by

$$
\begin{aligned}
& \operatorname{tr}\left[\Omega_{k+1 \mid k+1}\right] \\
= & \operatorname{tr}\left[\left(1+\epsilon_{3, k+1}\right) \Omega_{k+1 \mid k}-\left(1+\epsilon_{3, k+1}\right) \Omega_{k+1 \mid k} C_{k+1}^{T}\right. \\
& \times \sum_{i=1}^{N}\left(\Psi_{1, i}^{T} K_{i, k+1} \Psi_{2, i}\right)^{T} \\
& -\left(1+\epsilon_{3, k+1}\right) \sum_{i=1}^{N}\left(\Psi_{1, i}^{T} K_{i, k+1} \Psi_{2, i}\right) C_{k+1} \Omega_{k+1 \mid k} \\
& \left.+\sum_{i=1}^{N}\left(\Psi_{1, i}^{T} K_{i, k+1} \Psi_{2, i}\right) \Xi_{k+1} \sum_{i=1}^{N}\left(\Psi_{1, i}^{T} K_{i, k+1} \Psi_{2, i}\right)^{T}\right] \\
= & \operatorname{tr}\left[\left(1+\epsilon_{3, k+1}\right) \Omega_{k+1 \mid k}-\left(1+\epsilon_{3, k+1}\right) \Omega_{k+1 \mid k} C_{k+1}^{T}\right. \\
& \times \sum_{i=1}^{N}\left(\Psi_{1, i}^{T} K_{i, k+1} \Psi_{2, i}\right)^{T} \\
& -\left(1+\epsilon_{3, k+1}\right) \sum_{i=1}^{N}\left(\Psi_{1, i}^{T} K_{i, k+1} \Psi_{2, i}\right) C_{k+1} \Omega_{k+1 \mid k} \\
& \left.+\sum_{i=1}^{N}\left(\Psi_{1, i}^{T} K_{i, k+1} \Psi_{2, i}\right) \Xi_{k+1}\left(\Psi_{1, i}^{T} K_{i, k+1} \Psi_{2, i}\right)^{T}\right] .
\end{aligned}
$$

It should be noticed that, when deriving the second equation of (42), we have utilized the equation (41).

Next, take the partial derivative of $\operatorname{tr}\left[\Omega_{k+1 \mid k+1}\right]$ with respect to the estimator parameter matrix $K_{i, k+1}$ and set the derivative be zero. Moreover, according to Lemma 3, we arrive at

$$
\begin{aligned}
\frac{\partial \operatorname{tr}\left[\Omega_{k+1 \mid k+1}\right]}{\partial K_{i, k+1}}= & -2\left(1+\epsilon_{3, k+1}\right) \Psi_{1, i} \Omega_{k+1 \mid k} C_{k+1}^{T} \Psi_{2, i}^{T} \\
& +2 \Psi_{1, i} \Psi_{1, i}^{T} K_{i, k+1} \Psi_{2, i} \Xi_{k+1} \Psi_{2, i}^{T} \\
= & 0 .
\end{aligned}
$$

Note that $\Psi_{1, i} \Psi_{1, i}^{T}=I_{n \times n}$, then the optimal estimator parameter matrix is described by

$$
\begin{aligned}
K_{i, k+1}= & \left(1+\epsilon_{3, k+1}\right) \Psi_{1, i} \Omega_{k+1 \mid k} C_{k+1}^{T} \Psi_{2, i}^{T} \\
& \times\left(\Psi_{2, i} \Xi_{k+1} \Psi_{2, i}^{T}\right)^{-1},
\end{aligned}
$$

which is the same as that in (27). Consequently, the proof is complete.

Remark 4: Up to now, we have designed a varianceconstrained recursive state estimator for a class of discrete time-varying stochastic dynamical networks subject to uncertain inner coupling and signal quantization. In view of the information of the quantized measurements and the bounds of the inner coupling strength, the recursion of an optimal upper bound of the estimation error covariance is given, where the desired state estimation algorithm can be implemented via the solutions to two recursive matrix equations. In addition, the explicit form of the estimator parameter matrix is presented which can be easily computed at each sampling instant.

Remark 5: It is worthwhile to mention that the symmetric positive definite solutions of matrix equations (21) and (22) are dependent on the values of some given positive scalars $\epsilon_{1, k}, \epsilon_{2, k}, \epsilon_{3, k+1}, \epsilon_{4, k+1}$ and $\varepsilon_{k+1}$. Moreover, the feasibility of inequality constraint (23) affects the existence of the symmetric positive definite solutions of matrix equations (21) and (22). Hence, during the implementation, the proper $\varepsilon_{k+1}$ can be chose to ensure the feasibility of inequality constraint (23) and then to enhance the existence of the symmetric positive definite solutions of matrix equations (21) and (22).

\section{BOUNDEDNESS ANALYSIS}

In this section, we will propose a sufficient criterion to ensure that the resulting estimation error is exponentially mean-square bounded. To proceed, the following definition is needed which will be used in the subsequent developments.

Definition 1: [28] If there exist real scalars $\eta>0, \nu>0$ and $0<\vartheta<1$ such that

$$
\mathbb{E}\left\{\left\|\zeta_{k}\right\|^{2}\right\} \leq \eta \mathbb{E}\left\{\left\|\zeta_{0}\right\|^{2}\right\} \vartheta^{k}+\nu
$$

for each $k \geq 0$, then the stochastic process $\zeta_{k}$ is exponentially bounded in mean square sense.

According to Definition 1 and Theorem 1, the following theorem proposes a sufficient criterion to verify the exponential mean-square boundedness of the resulting estimation error.

Theorem 2: Consider the discrete time-varying stochastic complex networks (1)-(2) and the time-varying state estimator (6)-(7). If there exist real positive constants $\bar{a}, \underline{b}, \bar{b}, \bar{c}, \bar{w}, \underline{q}, \bar{q}$, $\bar{\tau}, \bar{\xi}_{1}$ and $\bar{\xi}_{2}$ satisfying the following conditions

$$
\begin{aligned}
& \left\|A_{i, k}\right\| \leq \bar{a}, \quad \underline{b} I \leq B_{i, k} B_{i, k}^{T} \leq \bar{b} I, \quad \underline{c} \leq\left\|C_{i, k}\right\| \leq \bar{c} \\
& \|\check{W}\| \leq \bar{w}, \underline{q} I \leq Q_{k} \leq \bar{q} I, \quad R_{k} \leq \bar{\tau} I \\
& \operatorname{tr}\left(\Xi_{k \mid k}\right) \leq \bar{\xi}_{1}, \operatorname{tr}\left(\Xi_{k+1 \mid k}\right) \leq \bar{\xi}_{2} \\
& \varrho=2 \vec{a}\left(1+\frac{\bar{c}^{2}}{\underline{c}^{2}}\right)^{2}<1
\end{aligned}
$$

where

$$
\begin{aligned}
\vec{a}= & \bar{a}^{2}+\left[\bar{w} \max _{i}\left(\frac{\underline{\gamma}_{i}+\bar{\gamma}_{i}}{2}\right)\right]^{2}, \\
\Xi_{k \mid k}= & \left(1+\epsilon_{2, k}\right) \Omega_{k \mid k}+\left(1+\epsilon_{2, k}^{-1}\right) \hat{x}_{k \mid k} \hat{x}_{k \mid k}^{T}, \\
\Xi_{k+1 \mid k}= & \left(1+\epsilon_{4, k+1}\right) \Omega_{k+1 \mid k}+\left(1+\epsilon_{4, k+1}^{-1}\right) \\
& \times \hat{x}_{k+1 \mid k} \hat{x}_{k+1 \mid k}^{T},
\end{aligned}
$$

then we can conclude that the estimation error is exponentially mean-square bounded.

Proof: Please see Appendix A.

Remark 6: So far, we have developed a new robust variance-constrained state estimation scheme for addressed 
time-varying stochastic complex networks with two factors contributing to the network complexities, that is, uncertain inner coupling and signal quantization. Accordingly, the corresponding information of these two factors has been explicitly reflected in Theorem 1. To be more specific, the matrices $\vec{\Gamma}$ and $\vec{\Gamma}$ characterize the uncertain inner coupling, and the scalars $\delta_{i}(i=1,2 \cdots, m)$ reflect the quantization effects. Besides, a sufficient condition has been proposed to analyze the boundedness issue of the resulting estimation error.

Remark 7: According to Theorem 2, it is not difficult to show that $\mathbb{E}\left\{\left\|\tilde{x}_{k+1 \mid k+1}\right\|\right\} \leq \sqrt{\frac{\phi}{\phi} \mathbb{E}\left\{\left\|\tilde{x}_{0 \mid 0}\right\|^{2}\right\} \vartheta^{k+1}+\frac{\varsigma \bar{\phi}}{1-\vartheta}}$, which implies that the upper bound of estimation error is dependent on the information of inner coupling strength, quantization parameters and the covariances of different noises. It can be seen that, the smaller the inner coupling error $\tilde{\Gamma}$, the smaller the parameter $\bar{r}$, the smaller the parameter $\varsigma$, and therefore the smaller final bound of the estimation error is obtained. Moreover, the bigger the quantization density $\chi^{(j)}$, the smaller the parameters $\bar{s}$ and $\bar{r}$, the smaller the parameter $\varsigma$, and therefore the smaller final bound of the estimation error is also obtained, which is consistent with the illustrations shown in the simulation. Moreover, a slightly smaller upper bound might be obtained if the system does not exist uncertain inner coupling and signal quantization because of the existence of different noises.

Remark 8: In this paper, the major aim is to reply the following three issues. (i) How to propose a proper model that is as comprehensive as possible to depict the time-varying complex dynamics networks? (ii) How to present an efficient and easy-to-implement estimation algorithm under the variance constraint to handle the effects induced by the uncertain inner coupling and signal quantization? (iii) How to evaluate the estimation algorithm performance with proper theoretical analysis criterion for the developed estimation method?

Remark 9: It is worthy to notice that some effective synchronization methods have been proposed in [16], [17] for time-invariant dynamics networks with communication constraints, where the missing channels have been modeled and discussed. Compared with the existing methods, major effort has been devoted to provide the variance-constrained recursive estimation algorithm for time-varying complex networks. In particular, our main results distinguish from existing ones in that: 1) we have made one of the first attempts to tackle both the uncertain inner coupling and the quantization errors for time-varying stochastic complex networks; 2) we have conducted comprehensive analysis on two important performance indices, namely, the estimation error variance and the estimation error bounds, for the addressed state estimation problem; and 3) we have proposed an effective recursive algorithm that corresponds to the time-varying nature of the underlying networks, thereby offers an attractive feature for online applications.

\section{An ILlustrative ExAmPle}

In this section, a simulation example is provided to illustrate the validity of the proposed state estimation algorithm under the variance constraint. The localization of multiple mobile robots is utilized to clarify the potential applicability of the proposed variance-constrained estimation method.

As analyzed in [21], consider the localization problem of multiple mobile robots via the visual tracking. The state could compose of the position and the orientation of the robot. In addition, the dynamics behaviour of each mobile robot is affected by other robots with the interaction and hence the coupling terms exist. When tracking a robot through a system equipped with sensors communicating via a (possibly wireless) network, the signal quantization might occur due to the bandwidth limitations of the communication channels. For realtime tracking, the system parameters would have to be timevarying and there exist different type noises. As such, our aim is to develop a new variance-constrained estimation method such that a locally optimal upper bound of the estimation error covariance is obtained, where both the uncertain inner coupling and the quantization errors are explicitly reflected in the main results.

Motivated by this background, we consider the discrete time-varying stochastic dynamical networks as in (1)-(2) with 3 nodes, and the coupling configuration matrix is given by

$$
W=\left[\begin{array}{lll}
0.3 & 0.2 & 0.2 \\
0.2 & 0.3 & 0.2 \\
0.2 & 0.2 & 0.3
\end{array}\right] .
$$

The related parameters are chosen by:

$$
\begin{aligned}
A_{1, k} & =\left[\begin{array}{cc}
0.55+0.1 \cos (2 k) & 0.35 \\
-0.6 & 0.75-0.1 \sin (k)
\end{array}\right], \\
A_{2, k} & =\left[\begin{array}{cc}
0.45+0.1 \sin (2 k) & -0.45 \\
0.75 & 0.7
\end{array}\right], \\
A_{3, k} & =\left[\begin{array}{cc}
0.85 & 0.75 \\
-0.35 & 0.35-0.1 \sin (6 k)
\end{array}\right], \\
B_{1, k} & =\left[\begin{array}{c}
0.6-0.1 \sin (5 k) \\
-0.6
\end{array}\right], \\
B_{2, k} & =\left[\begin{array}{c}
0.4-0.12 \cos (3 k)
\end{array}\right], B_{3, k}=\left[\begin{array}{c}
0.3 \\
0.5
\end{array}\right], \\
C_{1, k} & =\left[\begin{array}{cc}
1.96 & 1.3+0.1 \sin (2 k)
\end{array}\right], \\
C_{2, k} & =\left[\begin{array}{cc}
3+\cos (k) \sin (3 k) & 1.5
\end{array}\right], \\
C_{3, k} & =\left[\begin{array}{ll}
1.9+2 \cos (2 k) & 3.6+1.5 \sin (3 k)
\end{array}\right] .
\end{aligned}
$$

Here, the inner coupling matrix is $\Gamma=\operatorname{diag}\left\{\gamma_{1}, \gamma_{2}\right\}$, where the unknown coupling strength $\gamma_{i}(i=1,2)$ belongs to the interval $[0.3,0.5]$. Then, it is easy to obtain that $\bar{\Gamma}=\operatorname{diag}\{0.4,0.4\}$ and $\vec{\Gamma}=\operatorname{diag}\{0.1,0.1\}$.

In the simulation, the other parameters are selected as $x_{1,0}=\left[\begin{array}{ll}1.8 & 1.2\end{array}\right]^{T}, x_{2,0}=\left[\begin{array}{ll}1.2 & -1.6\end{array}\right]^{T}$, $x_{3,0}=\left[\begin{array}{ll}1.6 & 0.8\end{array}\right]^{T}, \hat{x}_{1,0 \mid 0}=\left[\begin{array}{ll}1.5 & -1.2\end{array}\right]^{T}, \hat{x}_{2,0 \mid 0}=$ $\left[\begin{array}{ll}1 & -2.3\end{array}\right]^{T}, \hat{x}_{3,0 \mid 0}=\left[\begin{array}{ll}-0.5 & 1.5\end{array}\right]^{T}, P_{1,0 \mid 0}=30 I_{2}$, $P_{2,0 \mid 0}=20 I_{2}, P_{3,0 \mid 0}=25 I_{2}, \epsilon_{1, k}=0.5, \epsilon_{2, k}=0.5, \epsilon_{3, k+1}=$ $0.1, \epsilon_{4, k+1}=1, \varepsilon_{k+1}=1 /\left(1.2 \lambda_{\max }\left(\tilde{\Lambda} R_{k+1} \tilde{\Lambda}\right)+0.5\right)$, $Q_{1, k}=Q_{3, k}=0.1, Q_{2, k}=0.2, R_{1, k}=R_{2, k}=0.2$ and $R_{3, k}=0.1$.

For the comparison purpose, the parameters of the logarithmic quantizer are chosen in the two cases, i.e., Case I: $u_{0}^{(1)}=$ $0.5, \chi^{(1)}=0.8$, Case II: $u_{0}^{(1)}=0.5, \chi^{(1)}=0.3$. As such, the newly developed variance-constrained state estimation method 
can be realized and the corresponding simulation results are plotted in Figs. 2-11. Figs. 2-7 depict one estimation result of the possible realizations, hence a single line refers to real state, the estimation, ideal measurement output and quantization signal, respectively. To be more specific, the real states of all network nodes and their estimations in two cases are plotted in Figs. 2-4. In Figs. 5-7, the ideal measurement outputs of all network nodes and the quantization signals are depicted. From the simulations in Figs. 2-7, it can be seen that the estimation results under Case I are better, which show that the proposed variance-constrained estimation method performs well when estimating the state of considered complex networks.

Secondly, we have provided the corresponding simulations in Figs. 8-10, where the average estimation error of each node after 500 simulation tests has been provided. From the simulations in Figs. 8-10, it can be observed that the smaller final bound of the estimation error can be obtained if the bigger quantization density $\chi^{(j)}$ is chose (i.e. Case I). That is to say, the estimation accuracy under the Case I is better compared with the one under the Case II, which is consistent with the assertion in Remark 7 and further illustrates that the newly proposed state estimation approach has a satisfactory estimation performance.

For the purpose of comparison, the traces of $P_{k+1 \mid k+1}$ and the traces of their upper bound $\Omega_{k+1 \mid k}$ under Cases I-II are depicted in Fig. 11. Again, according to the simulations, the larger the quantization density $\chi^{(j)}$, the better the estimation performance, which is in agreement with engineering practice. Overall, this paper makes the first attempt to tackle the variance-constrained estimation problem for time-varying stochastic complex networks with both the uncertain inner coupling and the quantization errors, which can be seen as the novelty/advantage of main results.
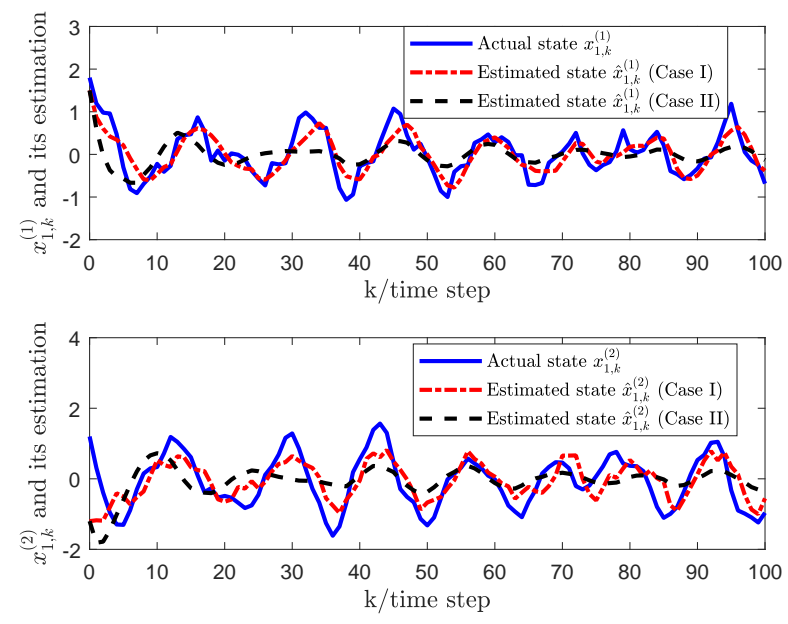

Fig. 2. Actual state $x_{1, k}$ and estimates under Cases I-II.

\section{CONCLusions}

In this paper, the variance-constrained state estimation problem has been discussed for a class of discrete time-varying
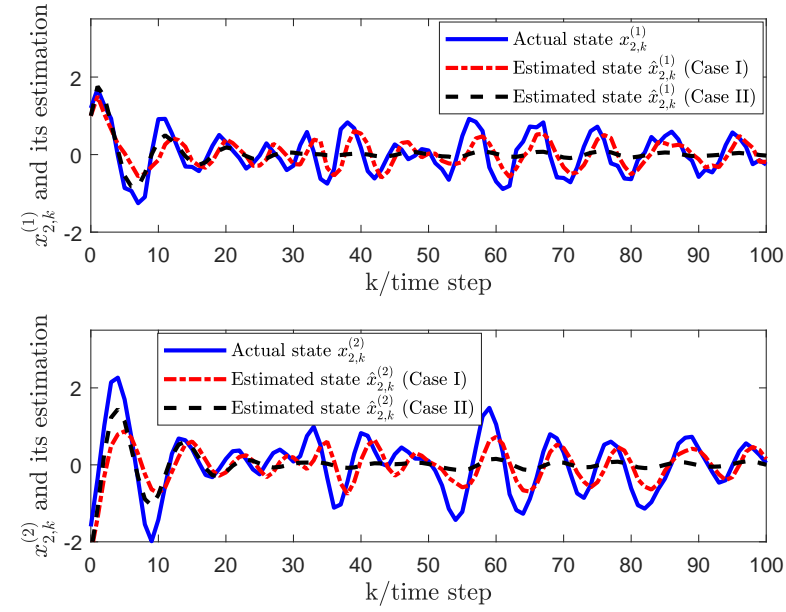

Fig. 3. Actual state $x_{2, k}$ and estimates under Cases I-II.
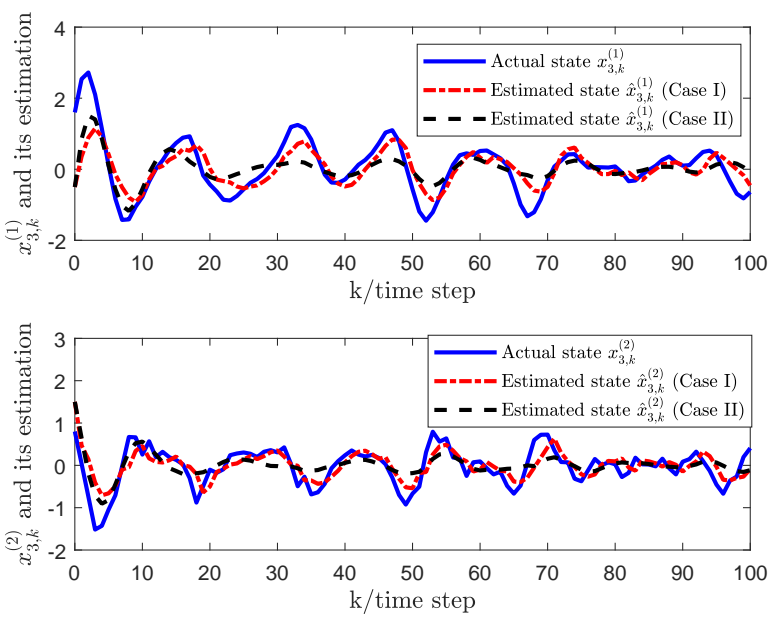

Fig. 4. Actual state $x_{3, k}$ and estimates under Cases I-II.

stochastic complex networks subject to uncertain inner coupling and quantization effects. A new state estimator has been constructed based on the quantized measurements, where the coupling configuration information of the networks has been properly reflected. In view of the intensive stochastic analysis technique, the recursion of the upper bound of the estimation error covariance has been established and then such an upper bound has been minimized at each time step by properly choosing the estimator gain matrix. Moreover, the detailed form of the estimator parameter matrix has been given. In addition, a sufficient condition has been derived to testify the boundedness of the estimation error. Finally, some simulations have been presented to show the validity of the optimal variance-constrained estimation algorithm proposed in this paper. The further research extensions include the investigation of variance-constrained recursive estimation problems for uncertain time-varying stochastic complex networks (e.g. with norm-bounded uncertainties) with/without the communication constraints as in [13], [43], where the desired results can be obtained in the near future. 


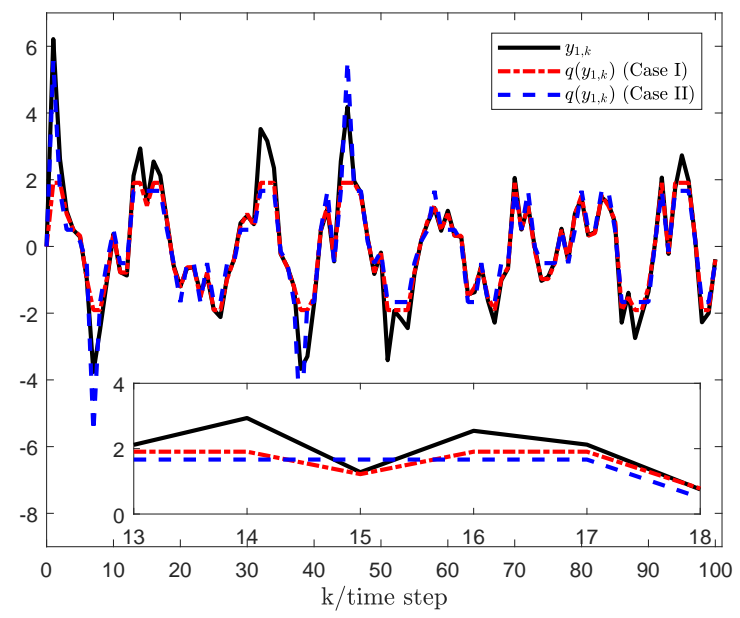

Fig. 5. The measurement output and quantization signal of $y_{1, k}$.

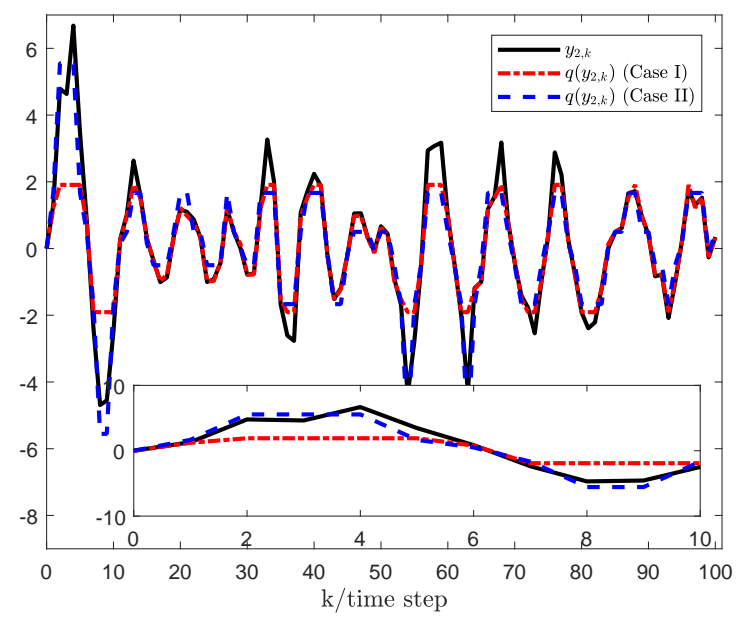

Fig. 6. The measurement output and quantization signal of $y_{2, k}$.

\section{APPENDIX}

\section{A. The Proof of Theorem 2}

Proof: Denoting $\mathcal{A}_{k}=A_{k}+W \otimes \bar{\Gamma}$ and noticing $\Pi_{k+1}=$ $\mathbb{I}-K_{k+1} C_{k+1}$, together with (10) and (11), we have

$$
\tilde{x}_{k+1 \mid k+1}=\Pi_{k+1} \mathcal{A}_{k} \tilde{x}_{k \mid k}+r_{k+1}+s_{k+1}
$$

where

$$
\begin{aligned}
& r_{k+1}=\Pi_{k+1}(W \otimes \tilde{\Gamma}) x_{k}-K_{k+1} \tilde{\Delta}_{k+1} C_{k+1} x_{k+1}, \\
& s_{k+1}=\Pi_{k+1} B_{k} \varpi_{k}-K_{k+1}\left(\mathbb{I}+\tilde{\Delta}_{k+1}\right) \nu_{k+1} .
\end{aligned}
$$

Based on (26) and (27), one has

$$
\begin{aligned}
\left\|K_{k+1}\right\| \leq & \max _{i}\left\|K_{i, k+1}\right\| \\
< & \max _{i} \|\left(1+\epsilon_{3, k+1}\right) \Psi_{1, i} \Omega_{k+1 \mid k} C_{k+1}^{T} \Psi_{2, i}^{T} \\
& \times\left[\left(1+\epsilon_{3, k+1}\right) \Psi_{2, i} C_{k+1} \Omega_{k+1 \mid k} C_{k+1}^{T} \Psi_{2, i}^{T}\right]^{-1} \| \\
< & \frac{\bar{c}}{\underline{c}^{2}}:=\bar{\kappa}
\end{aligned}
$$

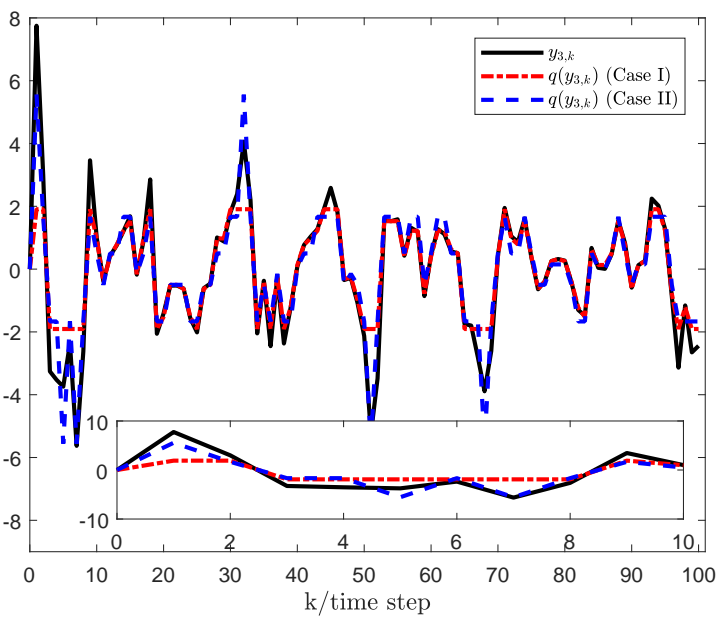

Fig. 7. The measurement output and quantization signal of $y_{3, k}$.

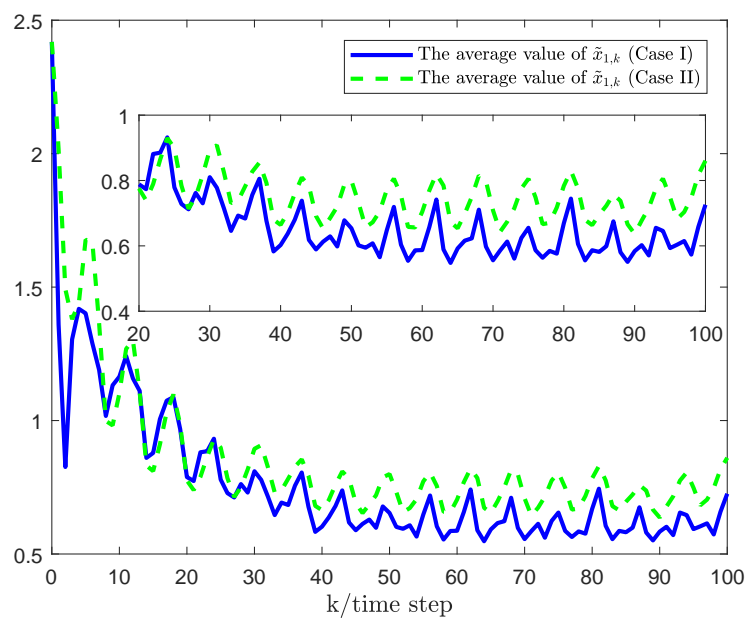

Fig. 8. The average error of $\tilde{x}_{1, k}$ after 500 iterations.

with $\underline{c}$ and $\bar{c}$ being defined in (46). Next, it is straightforward to see that

$$
\left\|\Pi_{k+1}\right\|=\left\|\mathbb{I}-K_{k+1} C_{k+1}\right\| \leq 1+\frac{\bar{c}^{2}}{\underline{c}^{2}}:=\bar{\pi} .
$$

Then, it follows from Lemma 1 and the properties of trace that

$$
\begin{aligned}
& \mathbb{E}\left\{r_{k+1}^{T} r_{k+1}\right\} \\
\leq & (1+\eta) \mathbb{E}\left\{x_{k}^{T}(W \otimes \tilde{\Gamma})^{T} \Pi_{k+1}^{T} \Pi_{k+1}(W \otimes \tilde{\Gamma}) x_{k}\right\} \\
& +\left(1+\eta^{-1}\right) \mathbb{E}\left\{x_{k+1}^{T} C_{k+1}^{T} \tilde{\Delta}_{k+1}^{T} K_{k+1}^{T}\right. \\
& \left.\times K_{k+1} \tilde{\Delta}_{k+1} C_{k+1} x_{k+1}\right\} \\
\leq & (1+\eta) \operatorname{tr}\left[\mathbb{E}\left\{x_{k} x_{k}^{T}\right\}(W \otimes \tilde{\Gamma})^{T} \Pi_{k+1}^{T} \Pi_{k+1}(W \otimes \tilde{\Gamma})\right] \\
& +\left(1+\eta^{-1}\right) \operatorname{tr}\left[\mathbb{E}\left\{x_{k+1} x_{k+1}^{T}\right\} C_{k+1}^{T} \tilde{\Delta}_{k+1}^{T} K_{k+1}^{T}\right. \\
& \left.\times K_{k+1} \tilde{\Delta}_{k+1} C_{k+1}\right] .
\end{aligned}
$$

Next, according to (46), (49)-(51) and $W \otimes \tilde{\Gamma}=\check{W} \check{\mathcal{F}} \check{\Gamma}$, one has

$$
\mathbb{E}\left\{r_{k+1}^{T} r_{k+1}\right\}
$$




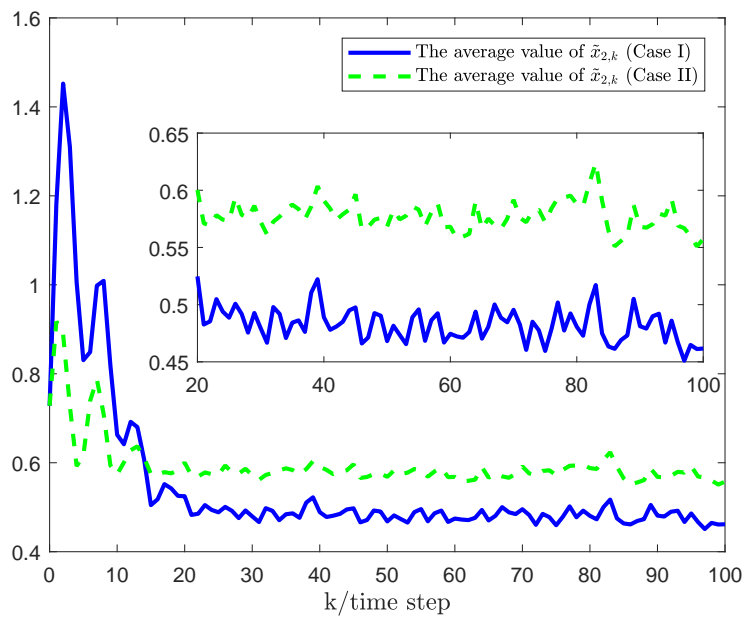

Fig. 9. The average error of $\tilde{x}_{2, k}$ after 500 iterations.

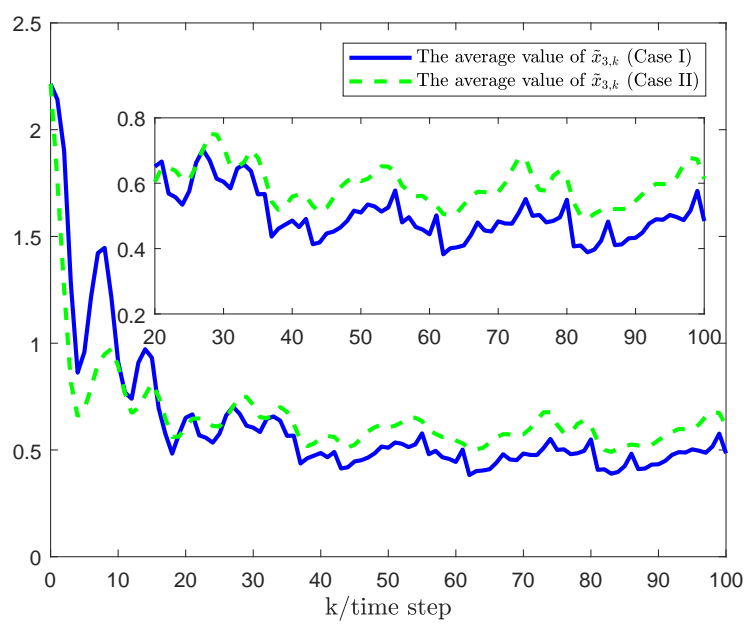

Fig. 10. The average error of $\tilde{x}_{3, k}$ after 500 iterations.

$$
\begin{aligned}
\leq & (1+\eta) \operatorname{tr}\left[\Xi_{k \mid k} \check{\Gamma}^{T} \check{\mathcal{F}}^{T} \check{W}^{T} \Pi_{k+1}^{T} \Pi_{k+1} \check{W} \check{\mathcal{F}} \check{\Gamma}\right] \\
& +\left(1+\eta^{-1}\right) \operatorname{tr}\left[\Xi_{k+1 \mid k} C_{k+1}^{T} \tilde{\Lambda}^{T} \tilde{\mathscr{F}}_{k+1}^{T} K_{k+1}^{T}\right. \\
& \left.\times K_{k+1} \tilde{\mathscr{F}}_{k+1} \tilde{\Lambda} C_{k+1}\right] \\
\leq & (1+\eta) \bar{\pi}^{2} \bar{w}^{2}\|\vec{\Gamma}\|^{2} \bar{\xi}_{1}+\left(1+\eta^{-1}\right) \bar{\kappa}^{2}\|\tilde{\Lambda}\|^{2} \bar{c}^{2} \bar{\xi}_{2} \\
:= & \bar{r}
\end{aligned}
$$

where $\eta>0$ is a scalar. Similarly, it is not difficult to see that

$$
\begin{aligned}
& \mathbb{E}\left\{s_{k+1}^{T} s_{k+1}\right\} \\
= & \mathbb{E}\left\{\varpi_{k}^{T} B_{k}^{T} \Pi_{k+1}^{T} \Pi_{k+1} B_{k} \varpi_{k}\right\} \\
& +\mathbb{E}\left\{\nu_{k+1}^{T}\left(\mathbb{I}+\tilde{\Delta}_{k+1}\right)^{T} K_{k+1}^{T} K_{k+1}\left(\mathbb{I}+\tilde{\Delta}_{k+1}\right) \nu_{k+1}\right\} \\
\leq & \bar{b} \bar{q} \bar{\pi}^{2}+2 \bar{\kappa}^{2}\left(1+\|\tilde{\Lambda}\|^{2}\right) \bar{\tau} \\
:= & \bar{s}
\end{aligned}
$$

Subsequently, we consider the following matrix equation with respect to the matrix $\Phi_{k}$ :

$$
\Phi_{k+1}=\Pi_{k+1} \mathcal{A}_{k} \Phi_{k} \mathcal{A}_{k}^{T} \Pi_{k+1}^{T}+B_{k} Q_{k} B_{k}^{T}+\delta_{0} I
$$

where $\Phi_{0}=B_{0} Q_{0} B_{0}^{T}+\delta_{0} I$ with $\delta_{0}>0$ being a scalar. Then, according to the above iterative matrix equation, it suffices to

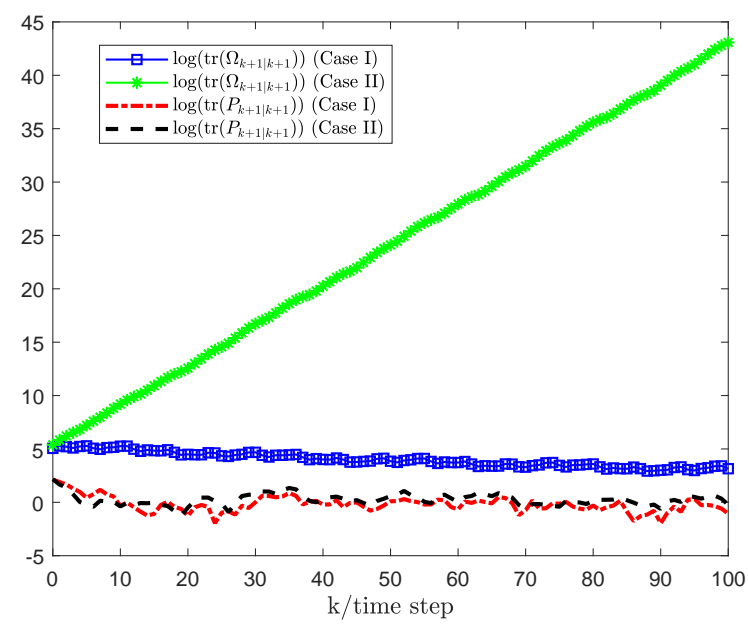

Fig. 11. The traces of $P_{k+1 \mid k+1}$ and their upper bound $\Omega_{k+1 \mid k+1}$.

see that

$$
\begin{aligned}
\left\|\Phi_{k+1}\right\| & \leq\left\|\Pi_{k+1}\right\|^{2}\left\|\mathcal{A}_{k}\right\|^{2}\left\|\Phi_{k}\right\|+\left\|B_{k} Q_{k} B_{k}^{T}\right\|+\left\|\delta_{0} I\right\| \\
& \leq \varrho\left\|\Phi_{k}\right\|+\bar{b} \bar{q}+\delta_{0}
\end{aligned}
$$

where $\varrho$ is defined as in (46). In addition, we can obtain the following inequality directly

$$
\left\|\Phi_{k}\right\| \leq \varrho^{k}\left\|\Phi_{0}\right\|+\left(\bar{b} \bar{q}+\delta_{0}\right) \sum_{i=0}^{k-1} \varrho^{i} .
$$

It follows from $\varrho<1$ that

$$
\begin{aligned}
\left\|\Phi_{k}\right\| & <\left\|\Phi_{0}\right\|+\left(\bar{b} \bar{q}+\delta_{0}\right) \sum_{i=0}^{\infty} \varrho^{i} \\
& =\left\|\Phi_{0}\right\|+\frac{\bar{b} \bar{q}+\delta_{0}}{1-\varrho} .
\end{aligned}
$$

On the other hand, we have

$$
\Phi_{k} \geq \delta_{0} I \text {. }
$$

In view of (57) and (58), there exist two positive scalars $\bar{\phi}$ and $\phi$ such that $\phi I \leq \Phi_{k} \leq \bar{\phi} I$ holds for all $k \geq 0$.

Subsequently, based on the matrix inversion lemma, we have

$$
\begin{aligned}
& \mathcal{A}_{k}^{T} \Pi_{k+1}^{T} \Phi_{k+1}^{-1} \Pi_{k+1} \mathcal{A}_{k}-\Phi_{k}^{-1} \\
= & \mathcal{A}_{k}^{T} \Pi_{k+1}^{T}\left(\Pi_{k+1} \mathcal{A}_{k} \Phi_{k} \mathcal{A}_{k}^{T} \Pi_{k+1}^{T}+B_{k} Q_{k} B_{k}^{T}+\varepsilon I\right)^{-1} \\
& \times \Pi_{k+1} \mathcal{A}_{k}-\Phi_{k}^{-1} \\
= & -\left[\Phi_{k}+\Phi_{k} \mathcal{A}_{k}^{T} \Pi_{k+1}^{T}\left(B_{k} Q_{k} B_{k}^{T}+\varepsilon I\right)^{-1} \Pi_{k+1} \mathcal{A}_{k} \Phi_{k}\right]^{-1} \\
= & -\left[\mathbb{I}+\mathcal{A}_{k}^{T} \Pi_{k+1}^{T}\left(B_{k} Q_{k} B_{k}^{T}+\varepsilon I\right)^{-1} \Pi_{k+1} \mathcal{A}_{k} \Phi_{k}\right]^{-1} \Phi_{k}^{-1} \\
\leq & -\bar{\alpha} \Phi_{k}^{-1}
\end{aligned}
$$

where $\bar{\alpha}=\left(1+\frac{2 \vec{a} \bar{\pi}^{2} \bar{\phi}}{\underline{q} \underline{b}}\right)^{-1}$ and $\vec{a}$ is defined in (47). Note that $\bar{\alpha} \in(0,1)$, then there exists a scalar $\epsilon>0$ satisfying $\vartheta=(1+\epsilon)(1-\bar{\alpha})<1$. Setting $V_{k}\left(\tilde{x}_{k \mid k}\right)=\tilde{x}_{k \mid k}^{T} \Phi_{k}^{-1} \tilde{x}_{k \mid k}$, it follows from (48) that

$$
\mathbb{E}\left\{V_{k+1}\left(\tilde{x}_{k+1 \mid k+1}\right) \mid \tilde{x}_{k \mid k}\right\}-(1+\epsilon) V_{k}\left(\tilde{x}_{k \mid k}\right)
$$




$$
\begin{aligned}
= & \mathbb{E}\left\{\left[\Pi_{k+1} \mathcal{A}_{k} \tilde{x}_{k \mid k}+r_{k+1}+s_{k+1}\right]^{T} \Phi_{k+1}^{-1}\right. \\
& \left.\times\left[\Pi_{k+1} \mathcal{A}_{k} \tilde{x}_{k \mid k}+r_{k+1}+s_{k+1}\right]\right\} \\
& -(1+\epsilon) \tilde{x}_{k \mid k}^{T} \Phi_{k}^{-1} \tilde{x}_{k \mid k} \\
= & \mathbb{E}\left\{\tilde{x}_{k \mid k}^{T}\left[\mathcal{A}_{k}^{T} \Pi_{k+1}^{T} \Phi_{k+1}^{-1} \Pi_{k+1} \mathcal{A}_{k}-(1+\epsilon) \Phi_{k}^{-1}\right] \tilde{x}_{k \mid k}\right\} \\
& +2 \mathbb{E}\left\{\tilde{x}_{k \mid k}^{T} \mathcal{A}_{k}^{T} \Pi_{k+1}^{T} \Phi_{k+1}^{-1} r_{k+1}\right\} \\
& +\mathbb{E}\left\{r_{k+1}^{T} \Phi_{k+1}^{-1} r_{k+1}\right\}+\mathbb{E}\left\{s_{k+1}^{T} \Phi_{k+1}^{-1} s_{k+1}\right\} .
\end{aligned}
$$

Then, in view of the Lemma 1 again, the following inequalities can be obtained:

$$
\begin{aligned}
& \mathbb{E}\left\{V_{k+1}\left(\tilde{x}_{k+1 \mid k+1}\right) \mid \tilde{x}_{k \mid k}\right\}-(1+\epsilon) V_{k}\left(\tilde{x}_{k \mid k}\right) \\
\leq & (1+\epsilon) \mathbb{E}\left\{\tilde{x}_{k \mid k}^{T}\left[\mathcal{A}_{k}^{T} \Pi_{k+1}^{T} \Phi_{k+1}^{-1} \Pi_{k+1} \mathcal{A}_{k}-\Phi_{k}^{-1}\right] \tilde{x}_{k \mid k}\right\} \\
& +\left(1+\epsilon^{-1}\right) \mathbb{E}\left\{r_{k+1}^{T} \Phi_{k+1}^{-1} r_{k+1}\right\} \\
& +\mathbb{E}\left\{s_{k+1}^{T} \Phi_{k+1}^{-1} s_{k+1}\right\} \\
\leq & -\bar{\alpha}(1+\epsilon) V_{k}\left(\tilde{x}_{k \mid k}\right)+\varsigma
\end{aligned}
$$

where $\varsigma=\left(1+\epsilon^{-1}\right) \frac{\bar{r}^{2}}{\underline{\phi}}+\frac{\bar{s}^{2}}{\underline{\phi}}$. Then, according to (60), we have

$$
\mathbb{E}\left\{V_{k+1}\left(\tilde{x}_{k+1 \mid k+1}\right) \mid \tilde{x}_{k \mid k}\right\} \leq \vartheta V_{k}\left(\tilde{x}_{k \mid k}\right)+\varsigma
$$

with $\vartheta=(1+\epsilon)(1-\bar{\alpha})$.

Finally, we arrive at

$$
\begin{aligned}
\mathbb{E}\left\{\left\|\tilde{x}_{k+1 \mid k+1}\right\|^{2}\right\} & \leq \frac{\bar{\phi}}{\underline{\phi}} \mathbb{E}\left\{\left\|\tilde{x}_{0 \mid 0}\right\|^{2}\right\} \vartheta^{k+1}+\varsigma \bar{\phi} \sum_{i=0}^{k} \vartheta^{i} \\
& \leq \frac{\bar{\phi}}{\bar{\phi}} \mathbb{E}\left\{\left\|\tilde{x}_{0 \mid 0}\right\|^{2}\right\} \vartheta^{k+1}+\varsigma \bar{\phi} \sum_{i=0}^{\infty} \vartheta^{i} \\
& =\frac{\bar{\phi}}{\underline{\phi}} \mathbb{E}\left\{\left\|\tilde{x}_{0 \mid 0}\right\|^{2}\right\} \vartheta^{k+1}+\frac{\varsigma \bar{\phi}}{1-\vartheta} .
\end{aligned}
$$

Therefore, it follows from Definition 1 that $\tilde{x}_{k \mid k}$ is exponentially mean-square bounded, which ends the proof of this theorem.

\section{REFERENCES}

[1] M. V. Basin, A. G. Loukianov, and M. Hernandez-Gonzalez, Joint state and parameter estimation for uncertain stochastic nonlinear polynomial systems, International Journal of Systems Science, vol. 44, no. 7, pp. 1200-1208, Jul. 2013.

[2] M. V. Basin, P. C. R. Ramirez, and F. Guerra-Avellaneda, Continuous fixed-time controller design for mechatronic systems with incomplete measurements, IEEE-ASME Transactions on Mechatronics, vol. 23, no. 1, pp. 57-67, Feb. 2018.

[3] M. V. Basin, P. Yu, and Y. B. Shtessel, Hypersonic missile adaptive sliding mode control using finite- and fixed-time observers, IEEE Transactions on Industrial Electronics, vol. 65, no. 1, pp. 930-941, Jan. 2018.

[4] S. Boccaletti, V. Latora, Y. Moreno, M. Chavez, and D.-U. Hwang, Complex networks: Structure and dynamics, Physics Reports, vol. 424, nos. 4-5, pp. 175-308, Feb. 2006.

[5] R. Caballero-Águila, I. García-Garrido, and J. Linares-Pérez, Information fusion algorithms for state estimation in multi-sensor systems with correlated missing measurements, Applied Mathematics and Computation, vol. 226, pp. 548-563, Jan. 2014.

[6] R. Caballero-Águila, A. Hermoso-Carazo, and J. Linares-Pérez, Distributed fusion filters from uncertain measured outputs in sensor networks with random packet losses, Information Fusion, vol. 34, pp. 7079, Mar. 2017.

[7] D. Ciuonzo, A. Aubry and V. Carotenuto, Rician MIMO channel- and jamming-aware decision fusion, IEEE Transactions on Signal Processing, vol. 65 , no. 15 , pp. 3866-3880, Aug. 2017.
[8] J. Dattorro, Convex optimization \& Euclidean distance geometry, Meboo Publishing, 2005.

[9] G. F. de Arruda, L. F. Costa, and F. A. Rodrigues, A complex networks approach for data clustering, Physica A: Statistical Mechanics and its Applications, vol. 391, no. 23, pp. 6174-6183, Dec. 2012.

[10] M. Fu and L. Xie, The sector bound approach to quantized feedback control, IEEE Transactions on Automatic Control, vol. 50, no. 11, pp. 1698-1711, Nov. 2005.

[11] F. Han, G. Wei, D. Ding, and Y. Song, Finite-horizon bounded $H_{\infty}$ synchronisation and state estimation for discrete-time complex networks: Local performance analysis, IET Control Theory \& Applications, vol. 11, no. 6, pp. 827-837, Apr. 2017.

[12] G. H. Hardy, J. E. Littlewood, and G. Pólya, Inequalities, 2nd edition, Cambridge University Press, 1988.

[13] J. Hu, Z. Wang, F. E. Alsaadi, and T. Hayat, Event-based filtering for time-varying nonlinear systems subject to multiple missing measurements with uncertain missing probabilities, Information Fusion, vol. 38, pp. 74-83, Nov. 2017.

[14] J. Hu, Z. Wang, and H. Gao, Joint state and fault estimation for uncertain time-varying nonlinear systems with randomly occurring faults and sensor saturations, Automatica, vol. 97, pp. 150-160, Nov. 2018.

[15] J. Hu, Z. Wang, S. Liu, and H. Gao, A variance-constrained approach to recursive state estimation for time-varying complex networks with missing measurements, Automatica, vol. 64, pp. 155-162, Feb. 2016.

[16] C. Huang, D. W. C. Ho, J. Lu, and J. Kurths, Pinning synchronization in T-S fuzzy complex networks with partial and discrete-time couplings, IEEE Transactions on Fuzzy Systems, vol. 23, no. 4, pp. 1274-1285, Aug. 2015.

[17] C. Huang, W. Wang, J. Cao, and J. Lu, Synchronization-based passivity of partially coupled neural networks with event-triggered communication, Neurocomputing, vol. 319, pp. 134-143, Nov. 2018.

[18] X. Jin, G. Yang, and W. Che, Adaptive pinning control of deteriorated nonlinear coupling networks with circuit realization, IEEE Transactions on Neural Networks and Learning Systems, vol. 23, no. 9, pp. 13451355, Sep. 2012.

[19] H. R. Karimi, Robust $H_{\infty}$ filter design for uncertain linear systems over network with network-induced delays and output quantization, Modelling Identification and Control, vol. 30, no. 1, pp. 27-37, 2009.

[20] Q. Li, B. Shen, J. Liang, and H. Shu, Event-triggered synchronization control for complex networks with uncertain inner coupling, International Journal of General Systems, vol. 44, no. 2, pp. 212-225, Feb. 2015.

[21] W. Li, Y. Jia, and J. Du, Variance-constrained state estimation for nonlinearly coupled complex networks, IEEE Transactions on Cybernetics, vol. 48, no. 2, pp. 818-824, Feb. 2018.

[22] X. Li and G. Yang, FLS-based adaptive synchronization control of complex dynamical networks with nonlinear couplings and state-dependent uncertainties, IEEE Transactions on Cybernetics, vol. 46, no. 1, pp. 171180, Jan. 2016

[23] J. Liu, F. Wu, L. Zhang, and S. Fei, Co-design of event generator and state estimator for complex network systems with quantization, Journal of Franklin Institute, vol. 353, no. 17, pp. 4565-4582, Nov. 2016.

[24] W. Lu, F. M. Atay, and J. Jost, Synchronization of discrete-time dynamical networks with time-varying couplings, SIAM Journal on Mathematical Analysis, vol. 39, no. 4, pp. 1231-1259, 2008.

[25] Z. Ning, L. Zhang, J. D. Rubio, and X. Yin, Asynchronous filtering for discrete-time fuzzy affine systems with variable quantization density, IEEE Transactions on Cybernetics, vol. 47, no. 1, pp. 153-164, Jan. 2017.

[26] M. Park, S. H. Lee, O. M. Kwon, and A. Seuret, Closeness-centralitybased synchronization criteria for complex dynamical networks with interval time-varying coupling delays, IEEE Transactions on Cybernetics, vol. 48, no. 7, pp. 2192-2202, Jul. 2018.

[27] R. Rakkiyappan, G. Velmurugan, J. N. George, J. Nicholas, and R. Selvamani, Exponential synchronization of Lur'e complex dynamical networks with uncertain inner coupling and pinning impulsive control, Applied Mathematics and Computation, vol. 307, pp. 217-231, Aug. 2017.

[28] K. Reif, S. Günther, E. Yaz, and R. Unbehauen, Stochastic stability of the discrete-time extended Kalman filter, IEEE Transactions on Automatic Control, vol. 44, no. 4, pp. 714-728, Apr. 1999.

[29] B. Shen, Z. Wang, D. Ding, and H. Shu, $H_{\infty}$ state estimation for complex networks with uncertain inner coupling and incomplete measurements, IEEE Transactions on Neural Networks and Learning Systems, vol. 24, no. 12, pp. 2027-2037, Dec. 2013.

[30] B. Shen, Z. Wang, and X. Liu, Bounded $H_{\infty}$ synchronization and state estimation for discrete time-varying stochastic complex networks over 
a finite horizon, IEEE Transactions on Neural Networks, vol. 22, no. 1, pp. 145-157, Jan. 2011.

[31] P. Shi, H. Wang, and C. Lim, Network-based event-triggered control for singular systems with quantizations, IEEE Transactions on Industrial Electronics, vol. 63, no. 2, pp. 1230-1238, Feb. 2016.

[32] K. Sivaranjani, R. Rakkiyappan, J. Cao, and A. Alsaedi, Synchronization of nonlinear singularly perturbed complex networks with uncertain inner coupling via event triggered control, Applied Mathematics and Computation, vol. 311, pp. 283-299, Oct. 2017.

[33] Y. Wang, T. Bian, J. Xiao, and C. Wen, Global synchronization of complex dynamical networks through digital communication with limited data rate, IEEE Transactions on Neural Networks and Learning Systems, vol. 26, no. 10, pp. 2487-2499, Oct. 2015.

[34] Y. Wang, L. Wang, W. Yang, and X. Wang, Quantization effects on complex networks, Scientific Reports, vol. 6, Artitle no. 26733, May 2016.

[35] Y. Wang, L. Xie, and C. E. de Souza, Robust control of a class of uncertain nonlinear systems, Systems \& Control Letters, vol. 19, no. 2, pp. 139-149, Aug. 1992.

[36] Z. Wu, P. Shi, H. Su, and J. Chu, Sampled-data exponential synchronization of complex dynamical networks with time-varying coupling delay, IEEE Transactions on Neural Networks and Learning Systems, vol. 24, no. 8, pp. 1177-1187, Aug. 2013.

[37] Y. Xia, L. Dai, W. Xie, and Y. Gao, Network-based data-driven filtering with bounded noises and packet dropouts, IEEE Transactions on Industrial Electronics, vol. 64, no. 5, pp. 4257-4265, May 2017.

[38] Y. Xu, R. Lu, H. Peng, K. Xie, and A. Xue, Asynchronous dissipative state estimation for stochastic complex networks with quantized jumping coupling and uncertain measurements, IEEE Transactions on Neural Networks and Learning Systems, vol. 28, no. 2, pp. 268-277, Feb. 2017.

[39] H. Yang, Y. Xu, and J. Zhang, Event-driven control for networked control systems with quantization and Markov packet losses, IEEE Transactions on Cybernetics, vol. 47, no. 8, pp. 2235-2243, Aug. 2017.

[40] L. Zhang, Y. Zhu, and W. Zheng, State estimation of discrete-time switched neural networks with multiple communication channels, IEEE Transactions on Cybernetics, vol. 47, no. 4, pp. 1028-1040, Apr. 2017.

[41] D. Zhang, Q. Wang, D. Srinivasan, H. Li, and L. Yu, Asynchronous state estimation for discrete-time switched complex networks with communication constraints, IEEE Transactions on Neural Networks and Learning Systems, vol. 29, no. 5, pp. 1732-1746, May 2018.

[42] H. Zhang, J. Hu, H. Liu, X. Yu, and F. Liu, Recursive state estimation for time-varying complex networks subject to missing measurements and stochastic inner coupling under random access protocol, Neurocomputing, vol. 346, pp. 48-57, Jun. 2019.

[43] L. Zou, Z. Wang, H. Gao, and X. Liu, Event-triggered state estimation for complex networks with mixed time delays via sampled data information: The continuous-time case, IEEE Transactions on Cybernetics, vol. 45, no. 12, pp. 2804-2815, Dec. 2015.

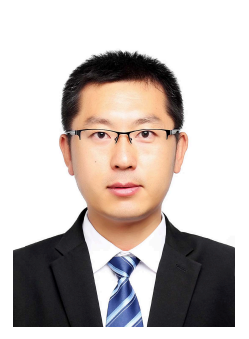

Jun Hu (M'14) received the B.Sc. degree in information and computation science and M.Sc. degree in applied mathematics from Harbin University of Science and Technology, Harbin, China, in 2006 and 2009, respectively, and the Ph.D. degree in control science and engineering from Harbin Institute of Technology, Harbin, China, in 2013.

From September 2010 to September 2012, he was a Visiting Ph.D. Student in the Department of Information Systems and Computing, Brunel University, U.K. From May 2014 to April 2016, he was an Alexander von Humboldt research fellow at the University of Kaiserslautern, Kaiserslautern, Germany. He is with the Department of Mathematics, Harbin University of Science and Technology, Harbin 150080, China, and also with the School of Engineering, University of South Wales, Pontypridd CF37 1DL, United Kingdom. His research interests include nonlinear control, filtering and fault estimation, time-varying systems and complex networks. He has published more than 40 papers in refereed international journals.

Dr. Hu serves as a reviewer for Mathematical Reviews, as an editor for IEEE Access, Neurocomputing, Journal of Intelligent and Fuzzy Systems, Neural Processing Letters, Systems Science and Control Engineering, and as a guest editor for International Journal of General Systems and Information Fusion.

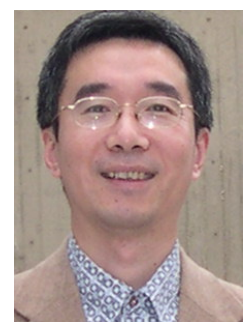

Zidong Wang (SM'03-F'14) was born in Jiangsu, China, in 1966. He received the B.Sc. degree in mathematics in 1986 from Suzhou University, Suzhou, China, and the M.Sc. degree in applied mathematics in 1990 and the Ph.D. degree in electrical engineering in 1994, both from Nanjing University of Science and Technology, Nanjing, China.

$\mathrm{He}$ is currently a Professor of Dynamical Systems and Computing in the Department of Computer Science, Brunel University London, U.K. From 1990 to 2002 , he held teaching and research appointments in universities in China, Germany and the UK. Prof. Wang's research interests include dynamical systems, signal processing, bioinformatics, control theory and applications. He has published more than 400 papers in refereed international journals. He is a holder of the Alexander von Humboldt Research Fellowship of Germany, the JSPS Research Fellowship of Japan, William Mong Visiting Research Fellowship of Hong Kong.

Prof. Wang serves (or has served) as the Editor-in-Chief for Neurocomputing, Deputy Editor-in-Chief for International Journal of Systems Science, and an Associate Editor for 12 international journals including IEEE Transactions on Automatic Control, IEEE Transactions on Control Systems Technology, IEEE Transactions on Neural Networks, IEEE Transactions on Signal Processing, and IEEE Transactions on Systems, Man, and Cybernetics-Part C. $\mathrm{He}$ is a Fellow of the IEEE, a Fellow of the Royal Statistical Society and a member of program committee for many international conferences.

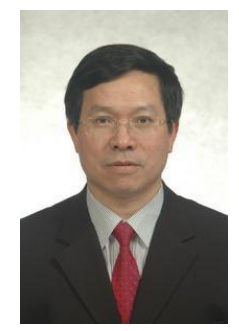

Guoping Liu (F'11) received the B.Eng and M.Eng degrees in automation from the Central South University, Changsha, China, in 1982 and 1985, respectively, and the Ph.D. degree in control engineering from the University of Manchester, Manchester, UK, in 1992. He is a professor with the University of South Wales, Pontypridd, U.K. and is a visiting professor with Wuhan University, China. His current research interests include networked multi-agent control systems, nonlinear system identification and control, advanced control of industrial systems, and multiobjective optimization and control. He has authored/co-authored over 200 journal papers and 10 books on control systems.

Prof. Liu is an editor-in-chief of the International Journal of Automation and Computing, IET Fellow and IEEE Fellow.

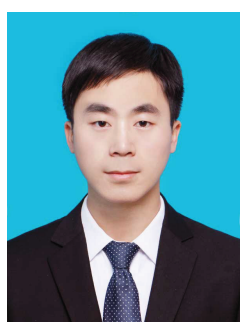

Hongxu Zhang received the B.Sc. degree in information and computation science and M.Sc. degree in mathematics from Harbin University of Science and Technology, Harbin, China, in 2014 and 2017, respectively. He is currently pursuing the $\mathrm{Ph} . \mathrm{D}$. degree in measuring and testing technologies and instruments at School of Measurement and Communication, Harbin University of Science and Technology, Harbin, China. His research interests include optimal state estimation and sliding mode control for complex dynamical systems.

$\mathrm{He}$ is an active reviewer for many international journals. 